\title{
Endiguement de la rivière Beas au droit du pont ferroviaire de l'embranchement Mirthal Mukerian-Pathankot
}

\author{
PAR H.L. UPPAL \\ DIRECTEUR DE L'IRRIGATION AND POWER RESEARCH INSTITUTE, PUNJAB, AMRITSAR \\ GAJINGER SINGH \\ ADJOINT AUX RECHERCHES \\ ET G.P. SHAHANI \\ INGËNIEUR EN CHEF DU GANGA BRIDGE PROJECT (MOKAMEH)
}

(English text, p. 873)

\begin{abstract}
Après le partage du territoire indou en 1947 et l'attribution à l'Inde des Etats de Jammu et de Kashmir, la construction d'une ligne de chemin de fer reliant directement Mukerian d̀ Pathankot fut décidée. Le projet comportait l'établissement d'un pont sur la rivière Beas à Mirthal. En cet endroit, la rivière forme de nombreux bras; comme la rive gauche est bien moins élevée que la rive droite, des difficultés surgirent pour canaliser la rivière et pour endiguer les crues. Il fallait décider si les bras de la rivière devaient être détournés vers le cours principal, ou si des passages devaient leur être ménagés individuellement à chaque croisement. L'examen approfondi de la question a exigé de
\end{abstract}

\begin{abstract}
nombreuses campagnes d'utudes sur place, de la rivière et de ses alentours.

Pour l'étude rationnelle des problèmes qui se présentaient, un modèle à grande échelle de la rivière, du pont projeté et des ouvrages attenants a été établi à la Station de Recherches Hydrauliques de Malakpur, à la suite de quoi un ensemble de travaux fut décidé, comprenant la construction d'une digue de dérivation détournant le bras du Barnai et d'une digue latérale de protection. L'idée de donner un patsage séparé aux divers afflnents fut abandonnée. Le projet ayant été entièrement réalisé, on a pu faire des observations sur place; leurs résultats ont concordé pleinement avec ceux qu'avait fourni le modèle.
\end{abstract}

\section{INTRODUCTION}

Avant le partage du Punjab, Pathankot était le terminus d'une voie large et, en même temps, la tête de ligne d'une voie étroite de la ligne ferroviaire iongeant la vallée du Kangra et desservant Kulu, Dharamsala et Dalhousie. Le trafic était principalement intense en direction de Lahore dans les deux sens; quant aux besoins de Jammu et de la vallée du Kashmir, les têtes de ligne de Rawalpindi et de Jammu y suffisaient. Le partage, en 1947, attribua ces deux têtes de ligne au Pakistan, mais l'attribution de Jammu et de Kashmir à l'Inde a détourné le trafic vers Delhi dans l'un et l'autre sens. Il fallait donc nécessairement établir une ligne plus directe vers Pathankot via Mukerian, réduisant ainsi de 44 miles $(71 \mathrm{~km})$ la distance séparant Jallundur de Pathankot.

Deux projets différents furent établis. Selon le premier, le tronçon de voie ferrée devait passer par Gurdaspur, enjambant les eaux de la Beas et du Chakki en un point dénommé Chechian, près de Gurdaspur. Selon le deuxième, le troncon à construire devait relier Pathankot à Mukerian directement. Après examen attentif, c'est celui-ci qui prévalut. Il comportait cependant la construction de très grands ponts, dont le principal devait franchir la Beas à Mirthal et l'autre le Chakki à Dangu, près de Pathankot; 


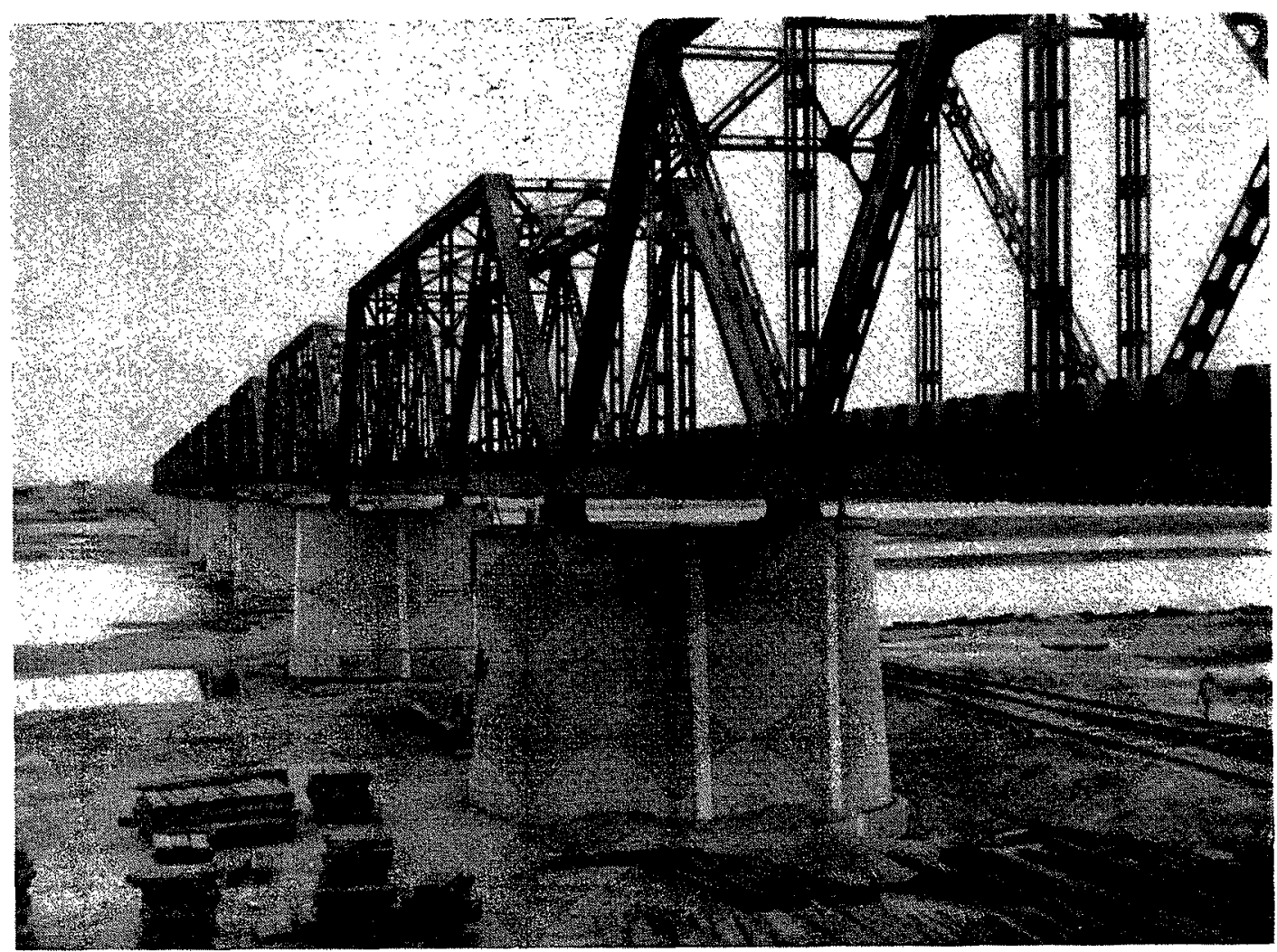

Le pont a Mirthal Mukerian en cours de construction. Bridge at Mirthal Mukerian under construction.

chacun d'eux comportait 14 travées de 150 pieds $(46 \mathrm{~m})$. La Beas était franchie en un point où elle forme de nombreux bras s'étalant dans une vaste vallée de 13.000 pieds $(4.000 \mathrm{~m})$ de largeur. Il fallait endiguer la rivière en un étroit chenal pour qu'elle soit enjambée par le pont, problème réellement difficile, qui a pu être résolu grâce à l'emploi de modèles fluviaux à grande échelle.

\section{La rivière Beas.}

Cette rivière prend naissance dans l'Himalaya, en un point dénommé Beas Kund, à 200 miles $(321 \mathrm{~km})$ de Pathankot. Elle traverse la chaîne de Dhaula Dhar à Larji et coule ensuite par monts et par vaux sur 390 miles $628 \mathrm{~km}$ avant de rejoindre la rivière Sutlej à Harike. Elle est le principal cours d'eau arrosant les vallées de Kangra et de Kulu, avec un bassin versant total de 7.122 miles carrés $\left(18.446 \mathrm{~km}^{2}\right)$. De nombreux torrents, comme le Binum, le Negal, le BanGanga, le Gaj, le Tara, le Chhaunch, etc., la rejoignent en cours de route. Son débit minimum est de $3.500 \mathrm{cs}\left(99 \mathrm{~m}^{3} / \mathrm{s}\right)$; il atteint $412.000 \mathrm{cu}$ secs, soit $11.666 \mathrm{~m}^{3} / \mathrm{s}$, aux plus fortes crues pendant la mousson.

La rivière débouche de la région montagneuse et pénètre dans la plaine à Reh, à 20 miles (32 km) en amont de Mirthal. Elle décrit un demi-cercle entre Reh et Nowshehra, à 8 miles $(13 \mathrm{~km})$ en aval de Mirthal, où elle dévie constamment vers la rive droite, faite d'argile dure, sans s'être détournée, semble-t-il, depuis plusieurs décennies. C'est une des raisons pour lesquelles on a choisi ce point.

En outre du bras principal qui se situe à l'extrême-droite, la rivière présente à Mirthal deux autres grands bras, le bras central et le bras du Barnai, situés respectivement à 2.000 pieds $(609 \mathrm{~m})$ et à 8.500 pieds $(2.590 \mathrm{~m})$ de la rive du côté de Mukerian et séparés par des îlots élevés, couverts d'un épais tapis d'herbe de Sarkanda. Ces îlots ne sont submergés qu'aux très grandes crues. A côté d'eux, on distingue, sur la figure 1, d'autres hauts-fonds et îlots.

Le bras de Barnai, très important aujourd'hui, n'existait pas en 1916 et la rivière coulait principalement dans le bras central. On avait creusé le chenal de Barnai uniquement pour servir à l'irrigation par submersion, sur une largeur de 15 pieds $(4,6 \mathrm{~m})$; peu à peu il devint le bras principal. Il se détache du bras principal en un point situé à 4 miles $(6,4 \mathrm{~km})$ en amont du talus du chemin de fer et forme aujourd'hui de nombreux méandres à pente rapide. Son lit présente une déclivité d'environ 40 pieds $(12 \mathrm{~m}$ ) entre le point de bifurcation et le croisement avec la voie ferrée. Ce bras continue à grrossir rapidement; 


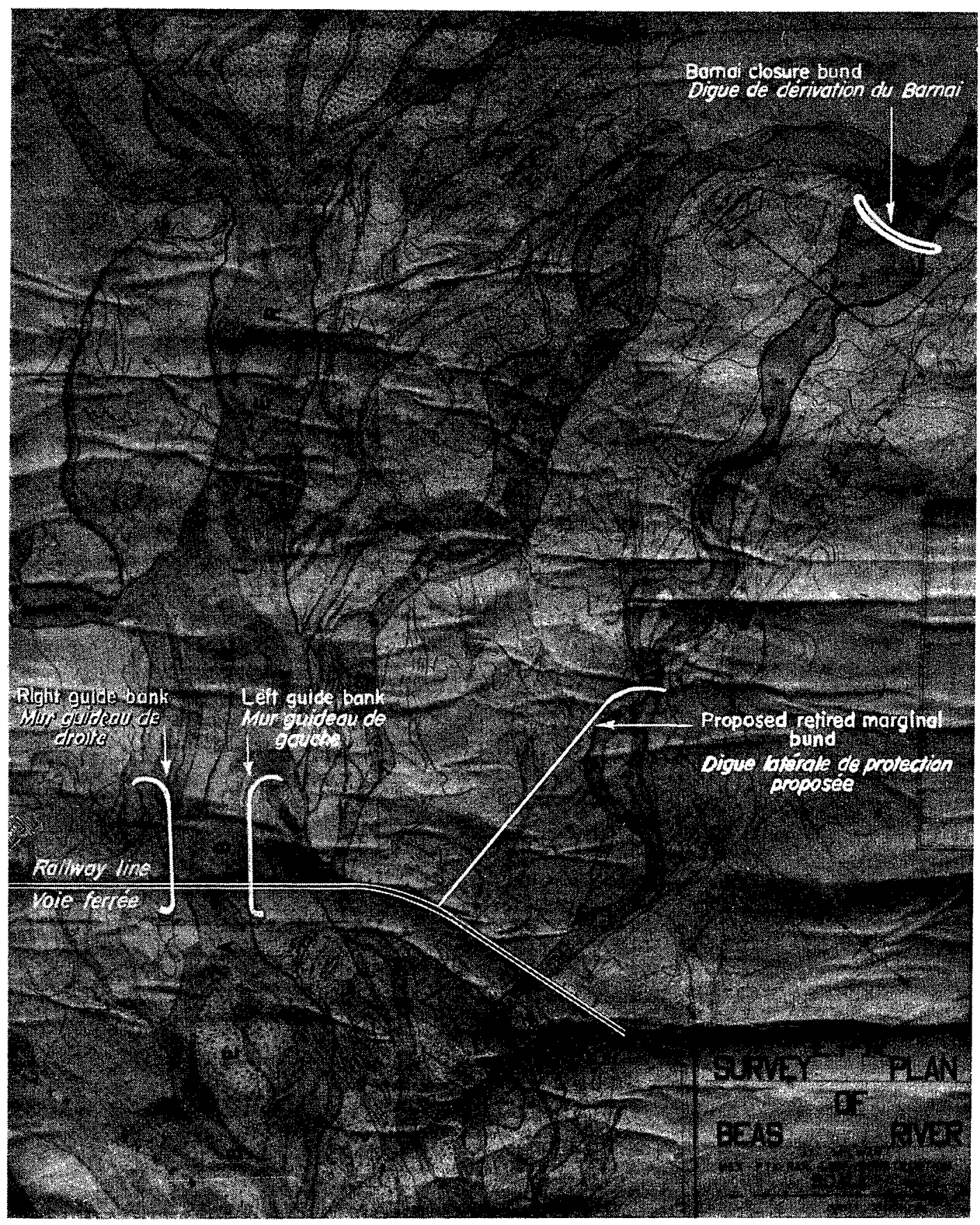

Fig. 1. - La rivière Beas, ses bras, ses hauts-fonds et les ilots de Sarkanda. Beas River, its creeks, shoals and Sarkanda belas.

son débit augmente d'année en année. Dans les deux années 1949 et 1950 , il a passé de $20.000 \mathrm{cs}$ $\left(560 \mathrm{~m}^{3} / \mathrm{s}\right)$ à environ 70.000 cusecs $\left(1.980 \mathrm{~m}^{3} / \mathrm{s}\right)$.

Quant à la rive gauche de la rivière Beas, à Mirthal, elle est bien moins haute que la rive droite et le sol s'incline vers Mukerian. Chaque fois done que la rivière monte, elle déborde sur la rive gauche et l'eau s'écoule vers Mukerian.
Matériaux du lit.

Le lit de la rivière est un mélange de sable et de galets ayant 3 à 4 pouces $(7,5$ à $10 \mathrm{~cm})$ de diamètre (fig. 2). Quant au lit du Barnai, il est formé de grains plus gros encore, le diamètre moyen des galets étant de 5 à 7 pouces $(13$ à $18 \mathrm{~cm}$ ).

Pente de la rivière. - Lat pente du lit, dans 


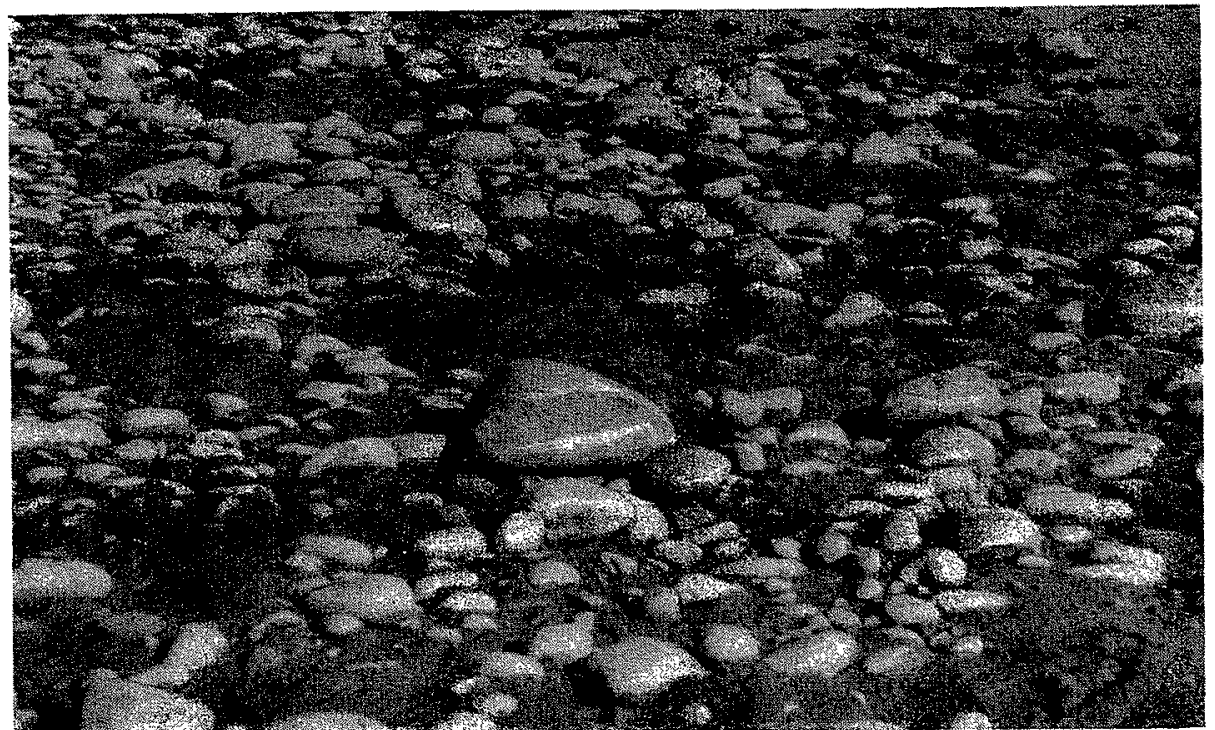

Fid. 2.

Le lit de la rivière Beas à Mirthal.

Bed of Beas River at Mirthal. le cours principal, est moins accentuée que celles des deux autres bras. Ces pentes sont: rivière principale $1: 1308$, bras central $1: 1093$; bras Barnai 1 : 865 .

A noter que le niveau du lit du Barnai, dans l'axe du pont, dépasse de 7,5 pieds $(2,30 \mathrm{~m})$ celui de la rivière principale, et de 5 pieds $(1,52 \mathrm{~m})$ celui du bras central.

Niveaux et débits de la rivière. - Aucune observation régulière du niveau et du débit ne fut faite à Mirthal, dans le passé. En 1950, quelques observations ont été effectuées par le Minis-

\section{TABLEAU I}

NTVEAUX DE L'EAY CORRESPONDANT A DIVERS DÉBITS OBSERVÉS SUR PLACE

AVANT LA CONSTRUCTION DU PONT

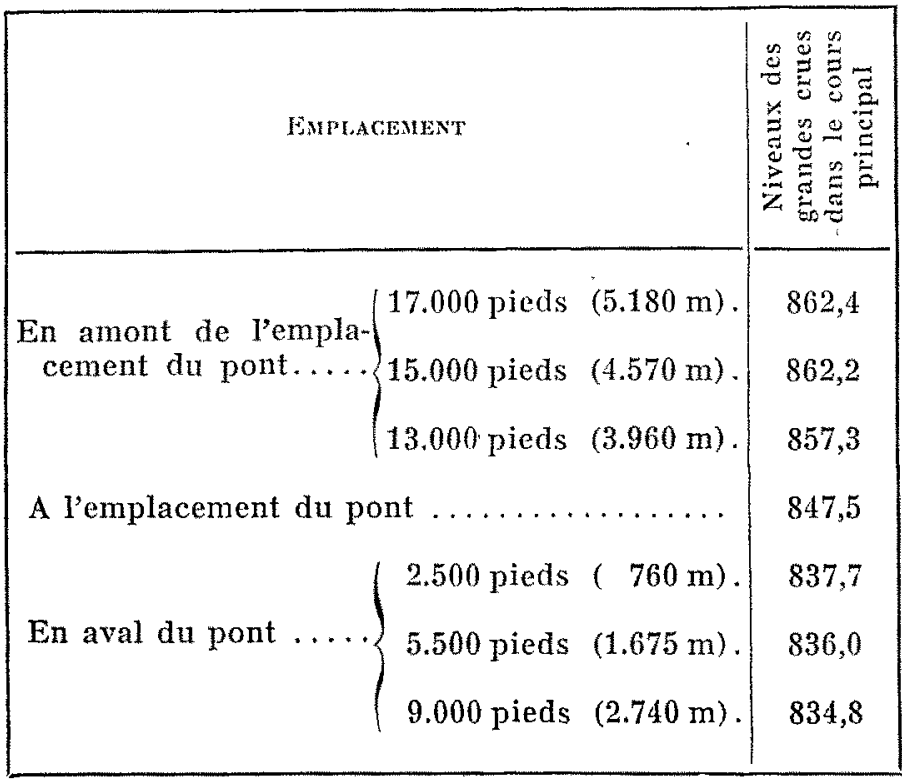

TABLEAU II

\begin{tabular}{|c|r|r|r|}
\hline \multirow{2}{*}{$\begin{array}{c}\text { Débit de la rivière } \\
\text { à Sujanpur Terrah }\end{array}$} & \multicolumn{2}{|c|}{$\begin{array}{c}\text { Cotes à l'emplacement du pont } \\
\text { Niveaud des grandes crues }\end{array}$} \\
\cline { 2 - 4 } & $\begin{array}{c}\text { au } \\
\text { bras no } 1\end{array}$ & $\begin{array}{c}\text { au } \\
\text { bras no }\end{array}$ & $\begin{array}{c}\text { au } \\
\text { bras } \mathrm{n}^{\circ} 3\end{array}$ \\
\hline $168.600 \mathrm{cs}\left(4.770 \mathrm{~m}^{3} / \mathrm{s}\right) \ldots$ & 832,2 & 837,82 & 837,66 \\
$250.000 \mathrm{cs}\left(7.090 \mathrm{~m}^{3} / \mathrm{s}\right) \ldots$ & 840,25 & 839,77 & 840,45 \\
$70.000 \mathrm{cs}\left(1.980 \mathrm{~m}^{3} / \mathrm{s}\right) \ldots$ & 836,75 & 837,00 & 838,05 \\
\hline
\end{tabular}

tère des Chemins de Fer; nous en donnons les chiffres (tabl. I). Pour quelques-unes, nous donnons aussi les débits correspondants, mesurés à Sujanpur Terrah (tabl. II).

\section{Projets d'endiguement de la rivière et de défense contre les crues.}

Le pont de chemin de fer devant être jetć pardessus le cours principal de la rivière, les deux autres bras : Barnai et Central, devaient être détournés et déversés dans le cours principal. Une autre solution consistait à ménager un passage spécial, à travers le talus, à chacun de ces bras. Ensuite, la construction dụ pont et le rétrécissement du cours d'eau qui en résulterait depuis 13.000 pieds $(3.950 \mathrm{~m})$ jusqu'aux 2.205 pieds $(670 \mathrm{~m})$ de largeur du thalweg représentant la longueur totale du pont, le fleuve se déploierait avec une profondeur légèrement plus grande jusqu'à ce qu'il ait aménagé son lit et retrouvé les conditions de régime. En plus de la détérioration du talus causée par l'écoulement parallèle, on 
aurait aussi à déplorer des dégâts à plusieurs villages ou villes et à de grandes étendues de cultures sur pied. Pour préserver le talus des effets de l'écoulement parallèle et pour mettre la campagne environnante à l'abri des inondations, on a eu l'idée de construire une digue latérale, de protection, hors d'atteinte de la rivière principale. Les crues de 1950 ont montré l'absolue nécessité de sa construction car de nombreux villages et une vaste étendue de cultures sur pied avaient été submergés, le talus avait été emporté sur une longueur de 2.000 pieds $(610 \mathrm{~m})$ et de nombreux ponts et ouvrages annexes furent endommagés. Ce malheur ne serait pas arrivé si la digue latérale de protection avait existé et si le Barnai avait été barré à son origine.

Trois projets furent établis :

1) Selon le premier, Ie bras Barnai devait être barré au franchissement de la voie ferrée et la digue latérale construite sur la rive gauche du Barnai, partant du point 48,000 chains $\left(^{*}\right)$ du talus du chemin de fer et terminée au sol à une éminence de cote 892,0 , près de Bakrarwan. Sa

(*) La chain représente 20,116 mètres longueur serait d'environ six miles et demi $(10,4 \mathrm{~km})$.

2) Selon le second projet, pour combattre la tendance manifeste du bras Barnai à gonfler tous les ans et pour éviter les sérieux dangers causés par les débordements de la rivière à travers les ouvertures placées près du pont, il fut considéré comme absolument nécessaire de barrer ce bras, soit à son origine, soit à son croisement avec la voie ferrée. Le barrage en ce dernier point n'était pas pratique, car il aurait créé un écoulement parallèle qui endommagerait encore le talus du chemin de fer. On s'est donc décidé à détourner le bras Barnai au point de bifurcation situé à quatre miles en amont, afin qu'il vienne renforcer le bras voisin et rejoigne ainsi la rivière principale; la construction d'une digue combinée de dérivation et de clôture du Barnai fut donc envisagée.

Pour empêcher l'eau accumulée de couler ver's Mukerian, d'endommager le talus et d'inonder en passant les terres basses, on résolut de construire une digue latérale de protection, partant du point 53.000 chains du talus et aboutissant à une éminence, de cote 861,0 , située sur un

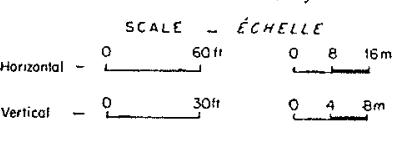

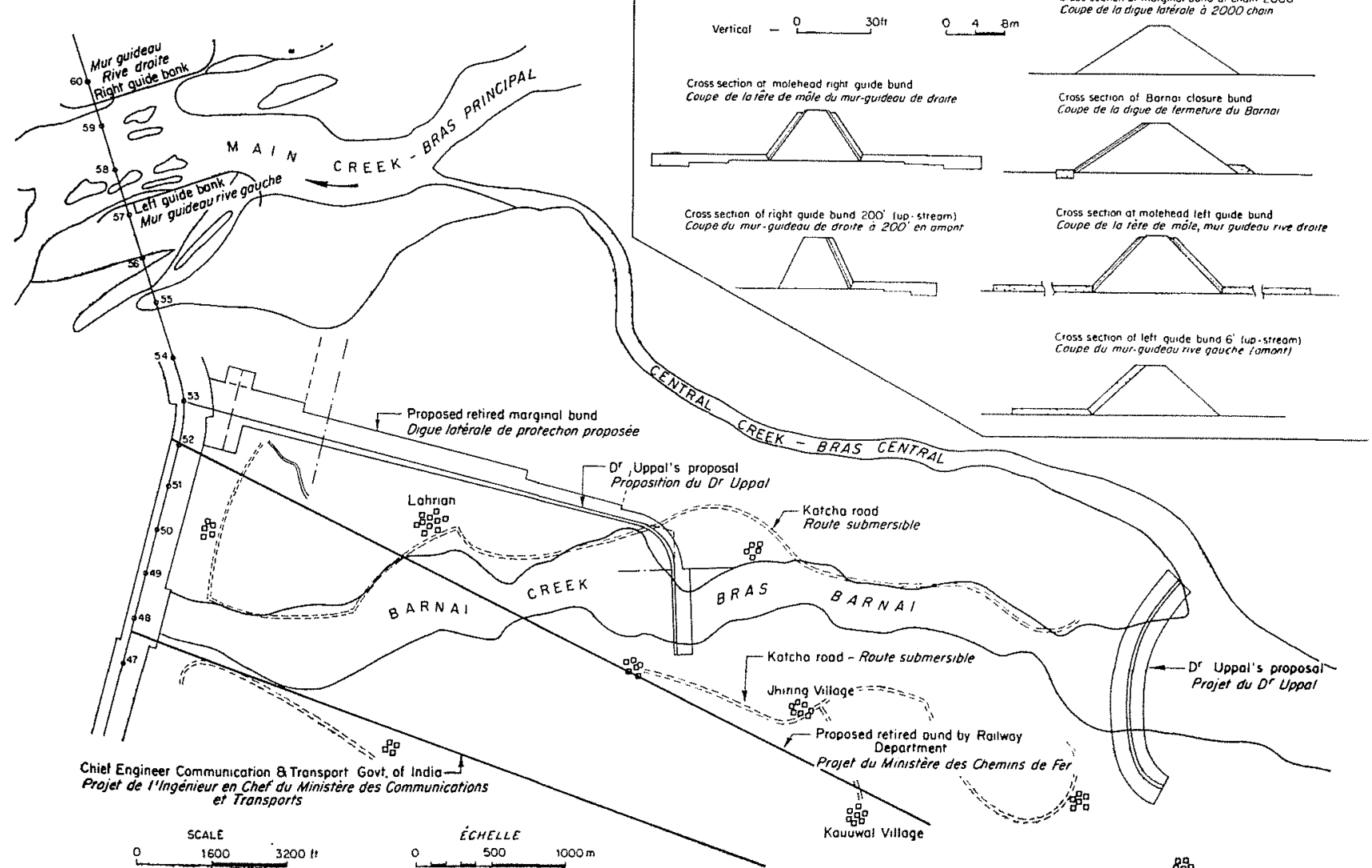

Fig. 3. - Les divers projets proposés pour l'endiguement de la rivière et la défense contre les crues.

Alternative proposals for the river training and flood control. 
îlot entre le Barnai et le bras central. Cette digue devrait avoir un mile et demi $(2,4 \mathrm{~km})$ de longueur, son sommet atteignant la cote 861,0 .

3) Un troisième projet prévoyait la construction de la digue latérale de protection atteignant plus ou moins le même alignement que selon le deuxième projet. Cependant, on devait la prolonger en amont, et après la traversée du Barnai entre les villages de Lahrian et Tanda, la rattacher à un point élevé, de cote 892,0 , près du village de Bakrarwan; sa longueur aurait été d'environ 6 miles $(9,6 \mathrm{~km})$. Mais, pour réussir, il fallait à tout prix barrer le bras Barnai, à son origine.

Ces différents projets sont indiqués sur la figure 3. Ils présentaient tous des problèmes difficiles et compliqués, qui furent étudiés au cours d'une série de conférences entre représentants de différents ministères; après discussions, la Direction des Chemins de Fer en communiqua les conclusions au Directeur de l'Institut de Recherches sur l'Irrigation et șur l'Energie du Punjab, à Amritsar, en vue de leur étude sur modèles à la Station de Recherches Hydrauliques de Malakpur.

\section{Les problèmes qui se posaient.}

Les problèmes suivants ont fait l'objet d'études sur modèles :

1) Comportement de la rivière et niveaux maxima de l'eau au pont, aux extrémités amont et aval des murs guideaux, contre le talus de la voie ferrée, dans les zones d'eau calme, etc., avec un débit maximum prévu à $420.000 \mathrm{cs}\left(11.900 \mathrm{~m}^{3} / \mathrm{s}\right)$.

2) Conditions de l'écoulement le long des murs guideaux et observation de l'entonnement au pont.

3) Clôture éventuelle du Barnai, bras majeur de la rivière, et son détournement vers le cours principal, ou bien parcours libre avec un pont séparé établi au croisement de ce bras avec la voie ferrée. Dans le premier cas, faudrait-il clore le bras au point où il quitte le bras central ou à son croisement avec la voie ferrée?

4) Emplacement et forme à donner à la digue de clôture et de dérivation du Barnai.

5) Recherche des meilleurs alignements et tracé de la digue latérale de protection.

\section{Les données.}

Les documents suivants ont pu être recueillis :

1) Plan topographique de la rivière sur une longueur de 4 miles $(6,4 \mathrm{~km})$ en amont du pont et 2,5 miles $(4 \mathrm{~km})$ en aval.
2) Profils de la rivière relevés tous les 500 pieds (152 m) sur une distance de 1,5 mile $(2,4 \mathrm{~km})$ et de tous les 1.000 pieds $(305 \mathrm{~m})$ sur 4 miles $(6,4 \mathrm{~km})$ côté amont. Du côté aval, sur 0,5 mile $(0,8 \mathrm{~km})$, l'espacement des relevés était de 500 pieds $(152 \mathrm{~m})$, et sur 2,5 milles $(4 \mathrm{~km})$, de 1.000 pieds (305 m).

3) Un avant-tprojet du pont et des ouvrages attenants.

4) Des sections et des plans topographiques des affluents Tara et Chhaunch.

5) Un ancien plan topographique de la région située à gauche du ponl.

6) Echantillons des matériaux du lit, valeurs isolées du débit et des niveaux observées à Mirthal en 1950 .

7) Vue aérienne de la rivière.

8) Une carte ancienne d'irrigation.

\section{Examen des données.}

L'examen des données qu'on possédait a conduit aux constatations suivantes:

I. - Entre le Barnai et le bras central, une crête s'étend sur 6.000 pieds $(1.830 \mathrm{~m})$ à partir du talus de la voie ferrée. Hormis la partie où son niveau et celui du sol sont peu élevés, elle dépasse n'importe lequel des niveaux de crue les plus hauts; elle n'est donc jamais submergée, même aux fortes crues.

II. - Le sol au-delà d'un mile $(1,6 \mathrm{~km})$ en amont du talus du chemin de fer est en pente de la gauche vers la droite; en un point situé à 4 miles $(1,4 \mathrm{~km})$ en amont du pont, la différence entre les niveaux de crue est de 24 pieds $(7,3 \mathrm{~m})$.

III. - A Mirthal, la rive gauche de la rivière est bien moins élevée que la droite. En temps de crue, l'eau inonde la rive gauche et toutes les terres basses jusqu'à Mukerian .

IV. - Le Barnai est plus important que le bras central. Le lit présente une pente de 35 à 40 pieds $(10$ à $12 \mathrm{~m}$ ) sur une largeur de $4,5 \mathrm{mi}-$ les $(7,2 \mathrm{~km})$. Le lit du bras principal a une pente de 15 à 17 pieds $(4,6$ à $5,2 \mathrm{~m})$ seulement, sur la même distance.

\section{Etude sưr modèle.}

LE Hopṫe. - Un modèle de la rivière Beas sur une longueur de 5 miles $(8 \mathrm{~km})$ en amont et 2 miles $(3,2 \mathrm{~km})$ en aval, s'étendant sur toute la largeur de la vallée et des bras et comprenant le pont du chemin de fer prévu et les ouvrages attenants, a été construit a la Station de Recherches Hydrauliques de Malakpur. Pour faconner le lit, on s'est servi d'un mélange de sable et de gravier d'un demi-pouce de diamè- 
Fic. 4.

Vue du modèle achevé.

liew of the completed model.

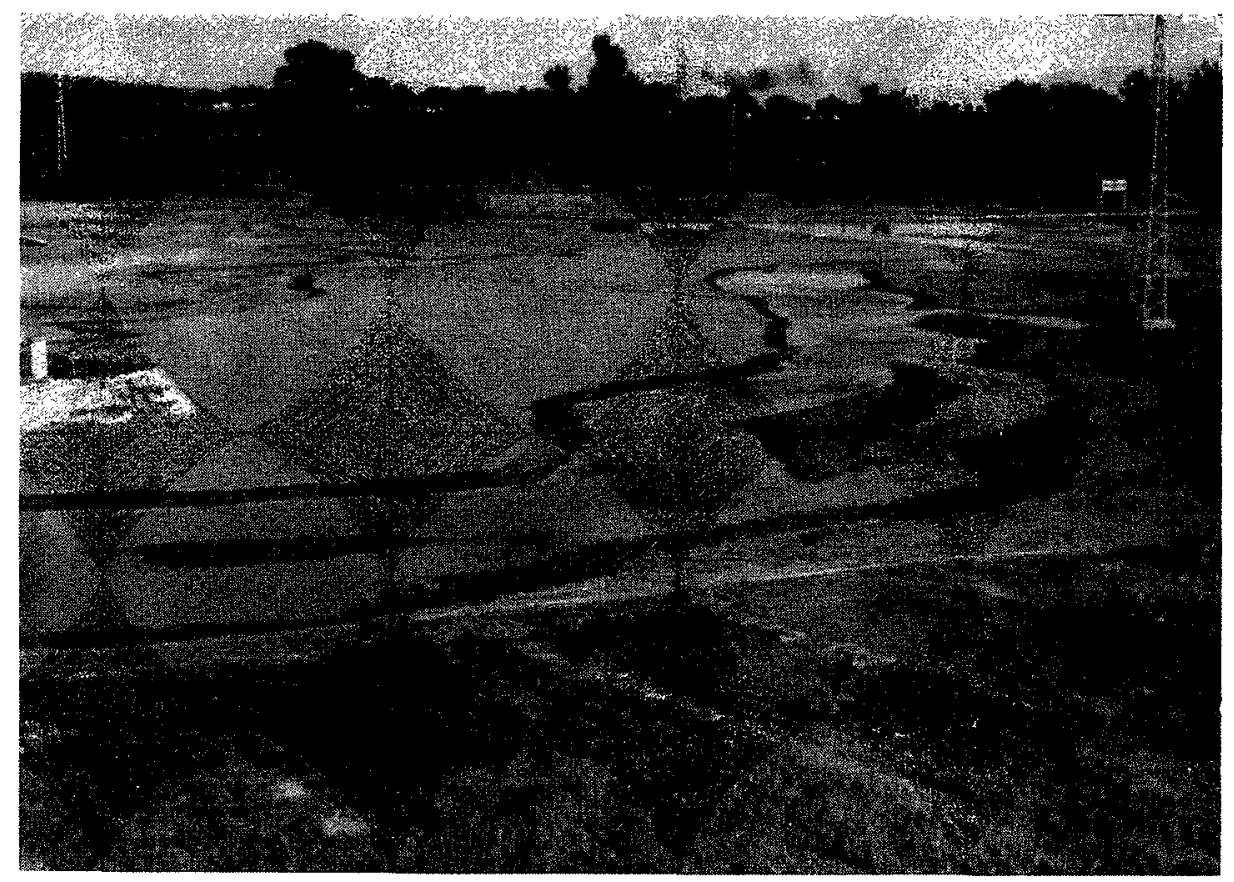

tre $(12,7 \mathrm{~mm})$. On a également reproduit les affluents les plus importants, comme le Tara et le Chhaunch, qui rejoignent la rivière sur le côté droit. En certains points importants, on a fixé sur le modèle des pointes de mesure donnant le niveau des eaux à divers débits. L'ensemble du modèle à l'état achevé est représenté sur la figure 4.

Les échelles adoptées étaient :

Modèle (dimensions horizonta-

les) : prototype......... $1: 90$

Modèle (dimensions verticales) :

prototype ........... $1: 30$

Distorsion . . . . . . . . . 3 .

Débits ............. $1: 14.788$

Superficie correspondant au mo-

dèle . . . . . . . $\left(25.083 \mathrm{~m}^{2}\right) 270,000 \mathrm{scr} . \mathrm{ft}$.

Débit du modèle.... $\left(0,8 \mathrm{~m}^{3} / \mathrm{s}\right) 28,4 \mathrm{cs}$

Sable et gravier utilisès $(1,27 \mathrm{~cm})$ diamètre 0.5 inch

OpÉRATIONS EFFECTUÉES SUR LE MODĖLE. - Le modèle n'a pu être éprouvé en l'absence des données nécessaires. Cependant, on admit qu'il n'était pas essentiel d'avoir des essais de contrôle pour cette étude.

Les études suivantes ont pu être effectuées :

1. Comportement de la rivière et détermination des niveaux maxima de l'eau au pont, aux extrémités amont et aval des murs-guideaux, le long du talus du chemin de fer, dans les zones d'eau morte, etc., avec le débit maximum adopté de $420.000 \mathrm{cs}\left(11.900 \mathrm{~m}^{3} / \mathrm{s}\right)$.
Le modèle fut essayé avec des débits variant entre $15.000 \mathrm{cs}\left(425 \mathrm{~m}^{3} / \mathrm{s}\right)$ et la valeur maximum prévue de $420.000 \mathrm{cs}(11.900 \mathrm{~m} / \mathrm{s})$, dont la majeure partie était fournie par la rivière Beas. Dans le cas d'un débit maximum de crue de $11.900 \mathrm{~m}^{3} / \mathrm{s}$, le Barnai véhiculait $75.000 \mathrm{cs}$ $\left(2.120 \mathrm{~m}^{3} / \mathrm{s}\right)$ et les niveaux maxima de 858,2 et 857,3 furent observés dans les zones d'eau morte, rive gauche et rive droite; un niveau maximum de 858,0 a été noté le long de la digue d'approche. Les niveaux maxima notés à l'anont et à l'aval du mur-guideau étaient 856,75 et 847,25 . Les différents niveaux enregistrés à tous les points importants sont notés dans le tableau III.

On a constaté que, jusqu'à un débit de $50.000 \mathrm{cs}\left(1.415 \mathrm{~m}^{3} / \mathrm{s}\right)$, la rivière ne débordait pas; mais elle débordait pour des débits plus forts et les eaux s'accumulaient contre le talus de la voie ferrée et y créaient des zones d'eaumorte en arrière des murs-guideaux. Ce débordement s'étendait sur des distances variables selon le débit (voir tableau IV).

$\mathrm{Ni}$ les îlots de Sarkanda, ni la crête entre le Barnai et le bras central n'étaient recouverts. Une partie seulement de la campagne, s'étendant jusqu'à 6.000 pieds $(1.830 \mathrm{~m})$ en amont du talus, était submergée. L'îlot entre le bras n" 2 et la rivière principale était en partie submergé.

2. Etude des conditions de l'écoulement le lon: des digues de guidage et observation de l'entonnement au pont.

Les sens et vitesses du courant ont été obser- 
TABLEAU III

NIVEAUX DE L'EAU POUR DIFFÉRENTS DÉBITS OBSERVÉS SUR LE MODĖLE

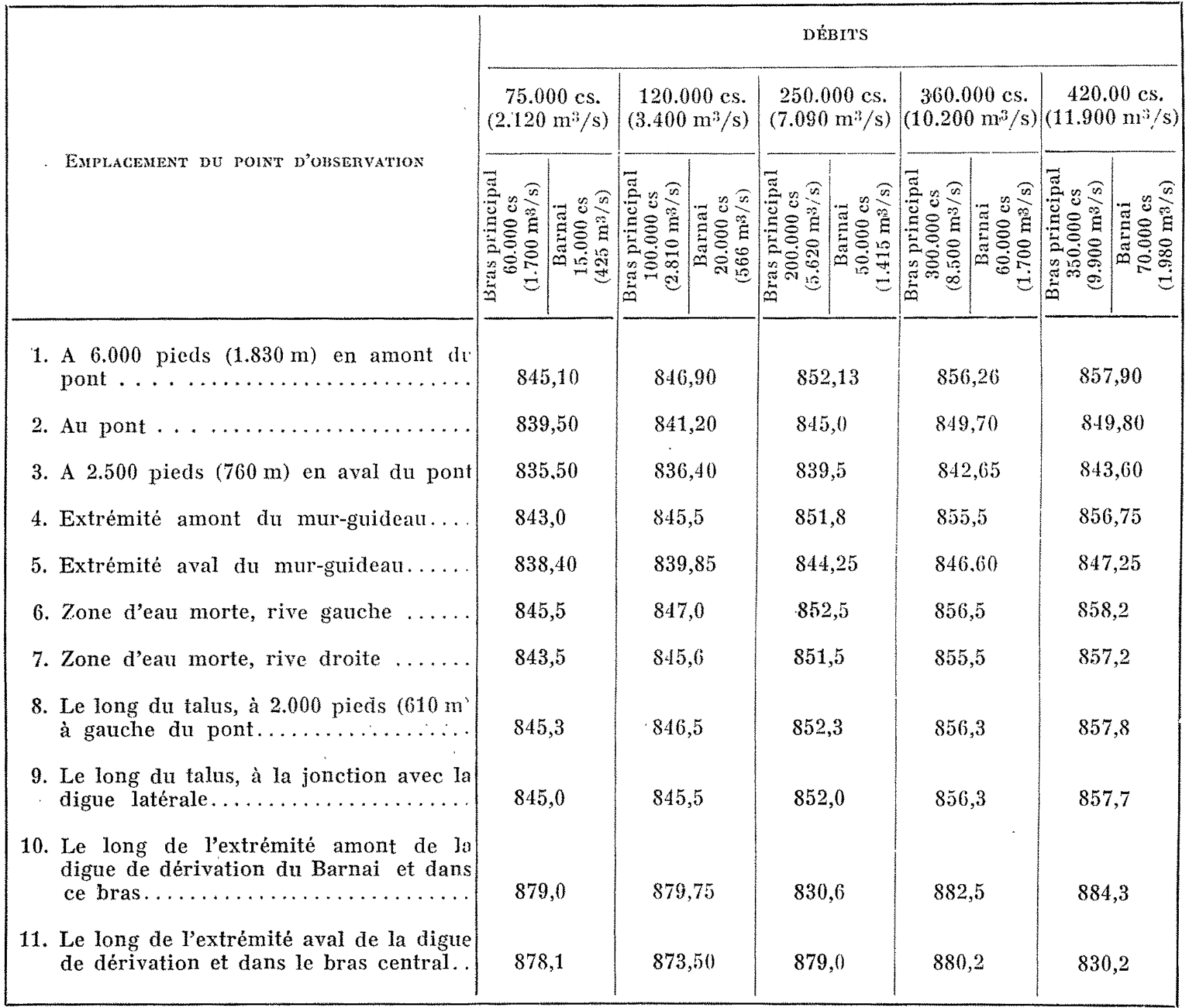

TABLEAU IV

VITESSES DE L'ÉCOULEMENT PARALLĖLE ET LONGUEUR DU REMOUS LE LONG DU TALUS DU GHEMIN DE FER

1. Vitesse max. de l'écoulement parallèle le long de la digue $\ldots \ldots \ldots \ldots \ldots \ldots \ldots \ldots$

2. Vitesse de l'écoulement autour de l'extrêmité amont du mur-guideau rive gauche.

3. Longueur du remous en amont du talus ferroviaire

\begin{tabular}{|c|c|c|c|}
\hline $\begin{array}{l}120.000 \mathrm{cs} \\
\left(3.400 \mathrm{~m}^{3} / \mathrm{s}\right)\end{array}$ & $\begin{array}{c}250.000 \mathrm{cs} \\
\left(7.090 \mathrm{~m}^{3} / \mathrm{s}\right)\end{array}$ & $\begin{array}{c}360.000 \mathrm{cs} \\
\left(10.200 \mathrm{~m}^{3} / \mathrm{s}\right)\end{array}$ & $\begin{array}{c}420.000 \mathrm{cs} \\
\left(11.900 \mathrm{~m}^{3} / \mathrm{s}\right)\end{array}$ \\
\hline $\begin{array}{l}6,50 \mathrm{ft} / \mathrm{sec} \\
(1,98 \mathrm{~m} / \mathrm{s})\end{array}$ & $\begin{array}{l}5,92 \mathrm{ft} / \mathrm{sec} \\
(1,80 \mathrm{~m} / \mathrm{s})\end{array}$ & $\begin{array}{l}1,08 \mathrm{ft} / \mathrm{sec} \\
(0,35 \mathrm{~m} / \mathrm{s})\end{array}$ & - \\
\hline $\begin{array}{l}4,38 \mathrm{ft} / \mathrm{sec} \\
(1,34 \mathrm{~m} / \mathrm{s})\end{array}$ & $\begin{array}{l}6,36 \mathrm{ft} / \mathrm{sec} \\
(1,94 \mathrm{~m} / \mathrm{s})\end{array}$ & $\begin{array}{l}6,24 \mathrm{ft} / \mathrm{sec} \\
(1,90 \mathrm{~m} / \mathrm{s})\end{array}$ & $\begin{array}{l}6,6 \mathrm{ft} / \mathrm{sec} \\
(2,01 \mathrm{~m} / \mathrm{s})\end{array}$ \\
\hline $\begin{array}{c}3,500 \mathrm{ft} \\
(1.065 \mathrm{~m})\end{array}$ & $\begin{array}{c}5.000 \mathrm{ft} \\
(1.524 \mathrm{~m})\end{array}$ & $\begin{array}{c}7.000 \mathrm{ft} \\
(2.133 \mathrm{~m})\end{array}$ & $\begin{array}{c}7.500 \mathrm{ft} \\
(2.286 \mathrm{~m})\end{array}$ \\
\hline
\end{tabular}


vés aussi bien le long des murs-guideaux que sur la rivière au droit du pont. L'écoulement était uniforme entre les murs (fig. 5 à 8). La vitesse maximum le long du mur-guideau était de l'ordre de $16,0 \mathrm{ft} / \mathrm{sec}$. $(4,88 \mathrm{~m} / \mathrm{s})$.

Mur-guideau rive gauche. - Aux faibles débits, aucune de ces digues ne subissait d'effet quelconque, mais aux forts débits le courant du bras principal vint donner contre le murguideau de gauche en un point situé à environ 450 pieds $(137 \mathrm{~m})$ à partir dù musoir amont (fig. 9) et produisit des effets désastreux (fig. 10). Toute la partie amont soit entre 2.300 pieds $(700 \mathrm{~m})$ et 3.000 pieds $(915 \mathrm{~m})$ à partir du talus

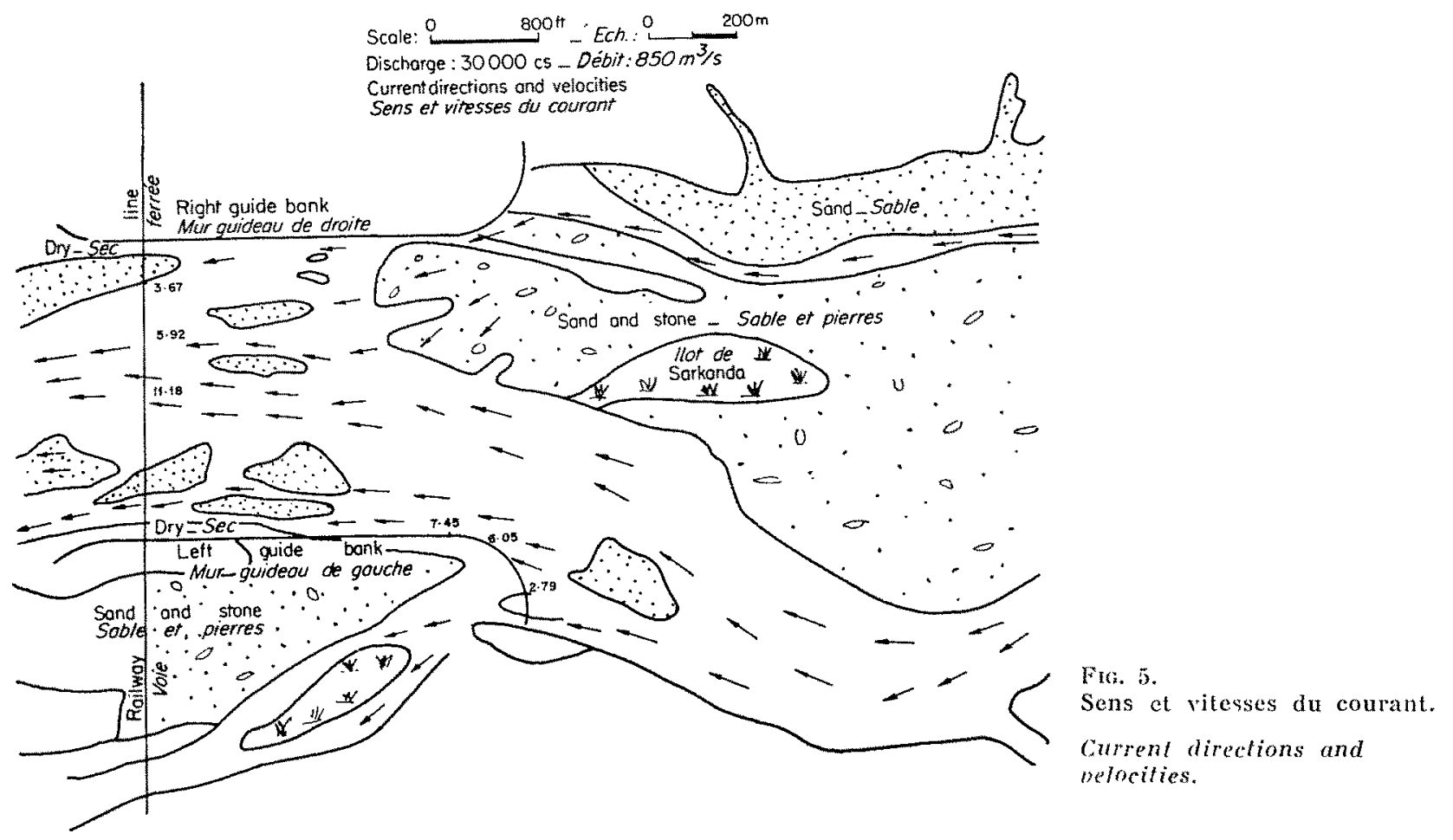

Fig. 6.

Sens et vitesses du courant.

current directions and velocities.

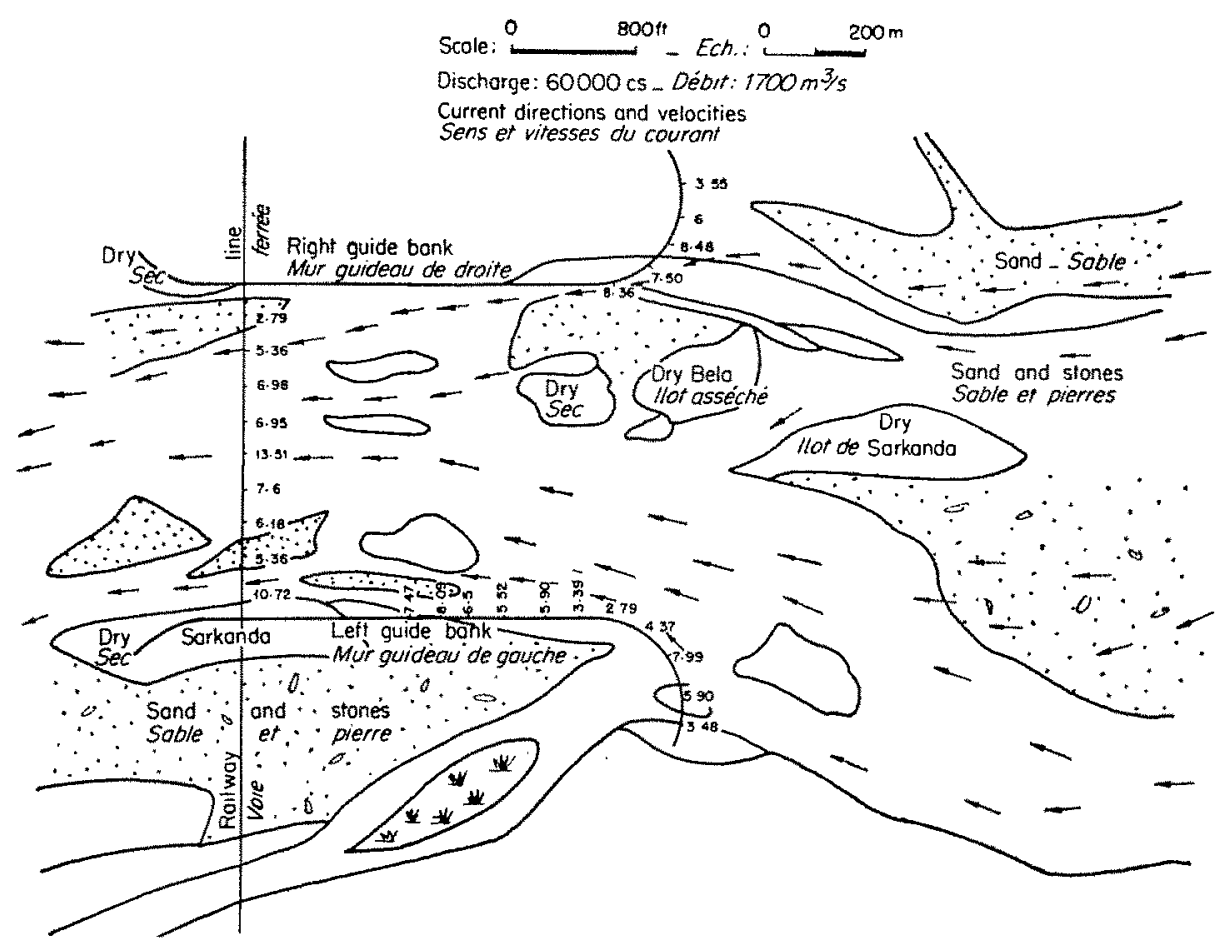


5. $16,1$.

Sens et vitesses du courant.

Citront directions and velocities.

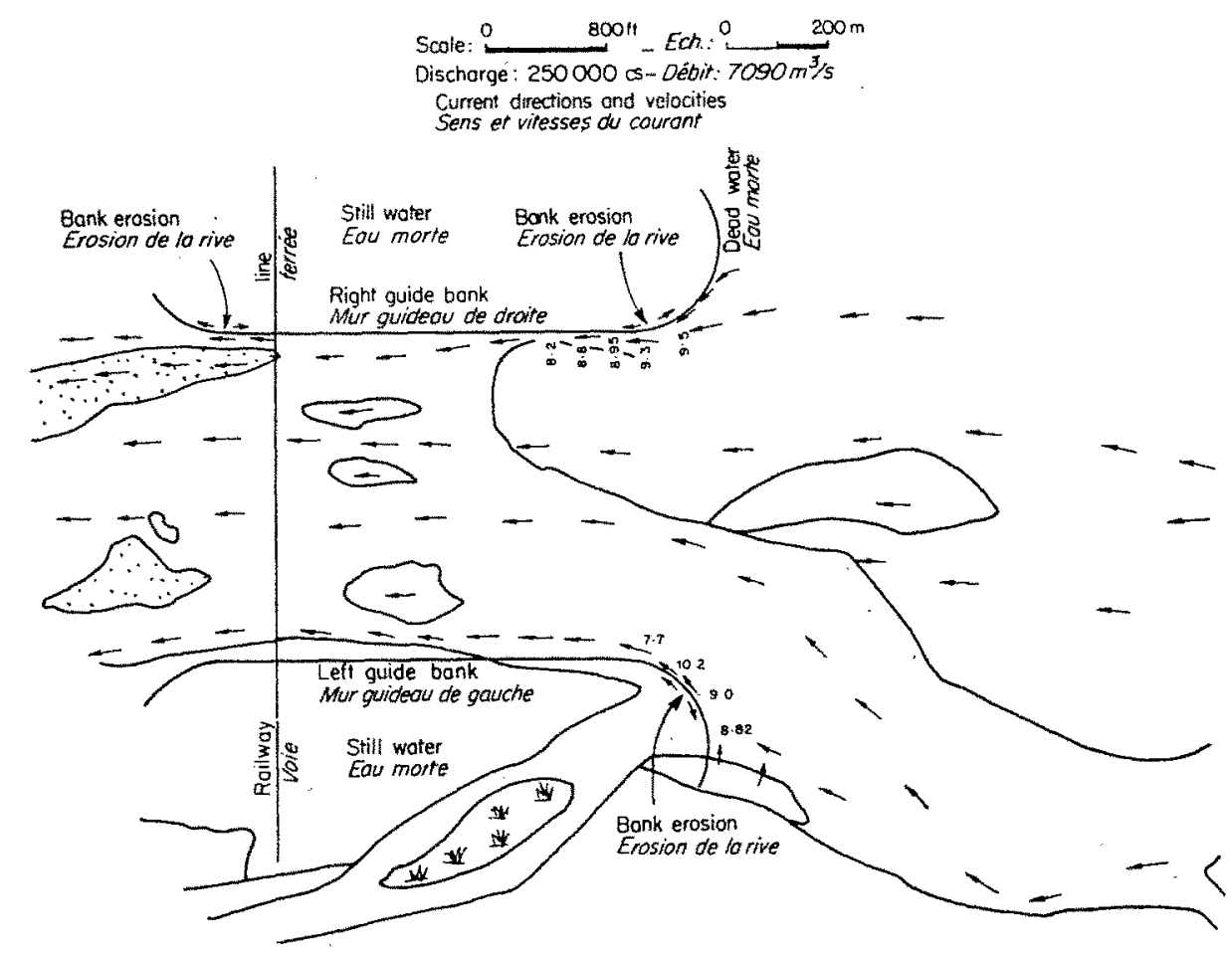

Scale : $\underbrace{800 \mathrm{ft}}_{\text {Discharge : } 420000 \mathrm{cs} \text { - Débit: }: 11900 \mathrm{~m}^{3} / \mathrm{s}}$

Current directions ond velocities
Sens of vitesses du courant

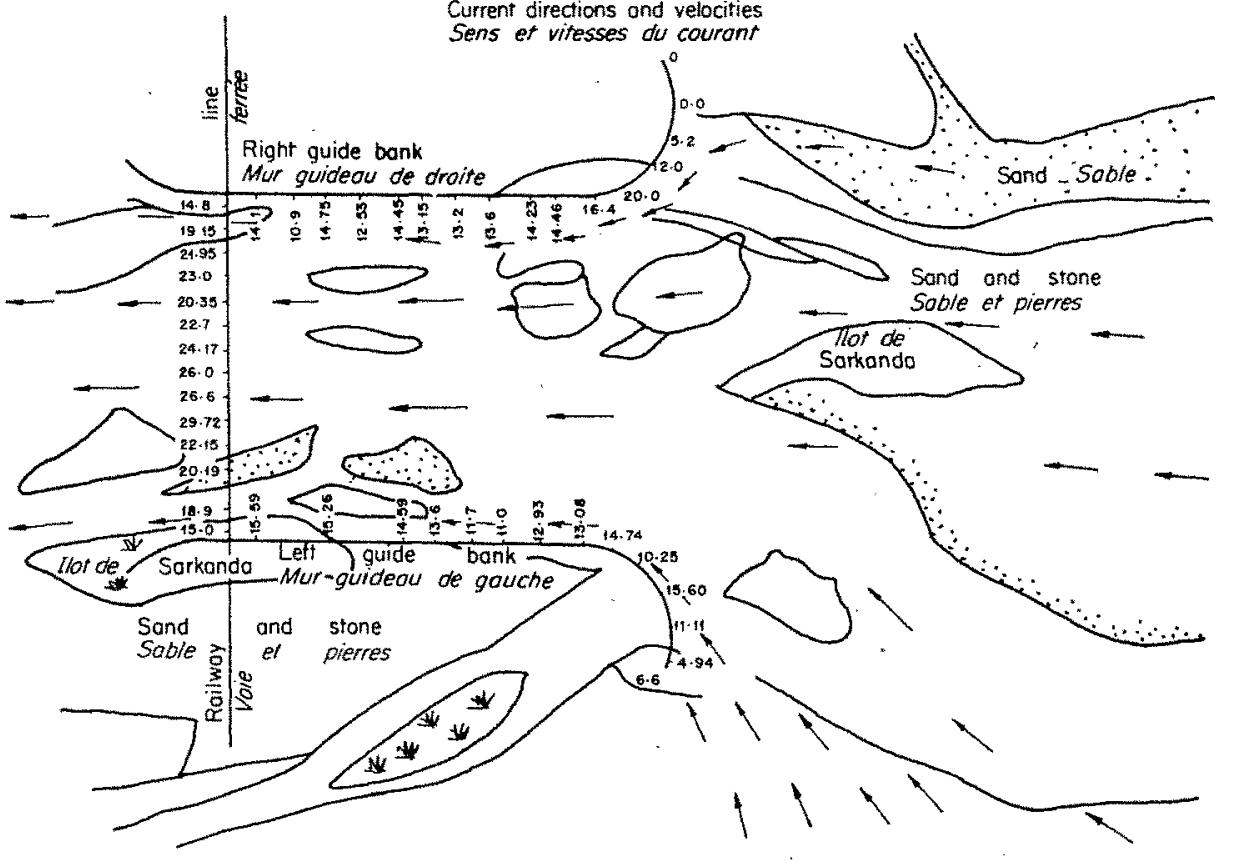

Fia. 8.

Sens at vitesses du cournt.

Current directions and velocities. eut à en subir l'assaut, particulièrement intense sur la partie courbe de la digue.

MUR-GUIDEAU RIVE DRoI'r. - Le courant principal rejoignait ce mur en aval de la partie Bell; sa partie amont baignait constamment dans une zone d'eau calme. La partie Bell du mur-guideau intervenait uniquement quand il $y$ avait syn- chronisme entre les crues de la rivière Beas et celles de son affluent le Chhaunch. Il se produisait en cet endroit un afflux d'eau de deux pieds plutòt excessif, étant donné la rapidité du flot.

3. Il s'agissait de décider si le Barnai, bras majeur de la rivière, devait être barré et détourné vers la rivière principale, ou si l'on 
Fig. 9.

Le bras principal heurtant la partie courbe du mur-guideau de la rive gauche.

Main current hits at the curved portion of left guide bank.

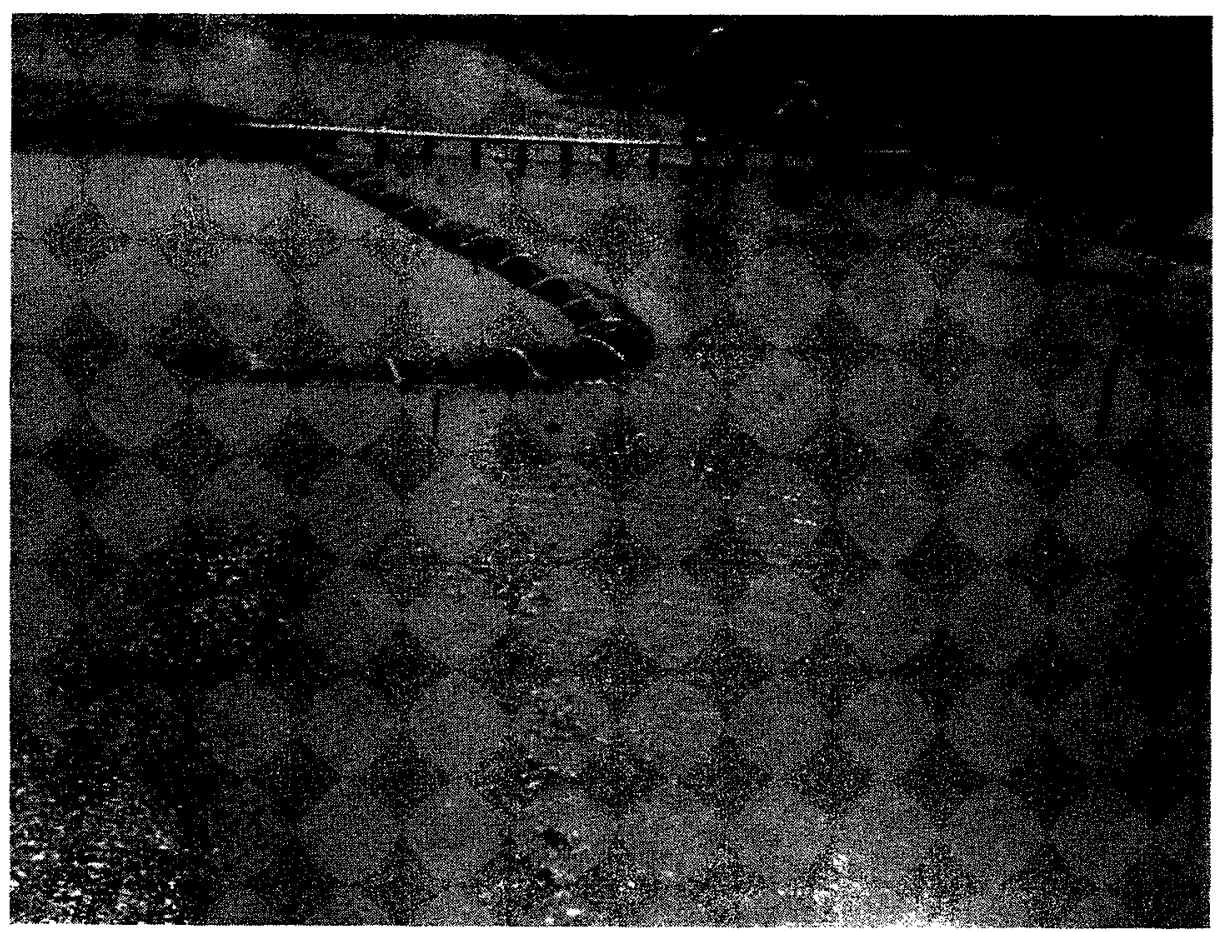

devait le laisser libre de couler en lui affectant un pont individuel, à son croisement avec la voie ferrée. S'il était à barrer, devait-on le faire au point où il se sépare du bras central, ou bien à son croisement avec la voie ferrée?

Le bras Barnai s'enflait d'année en année, et cette tendance était confirmée sur le modèle éga- lement; elle empêchait de déterminer exactement les dimensions des ouvertures à percer dans le talus pour que l'eau de ce bras puisse librement s'écouler. De plus, en présence du danger d'irruption de la rivière à travers ces nouvelles ouvertures supplémentaires, il n'a pas été jugé bon d'entreprendre d'importantes percées près du grand pont. Une grande ouverture, pratiquée

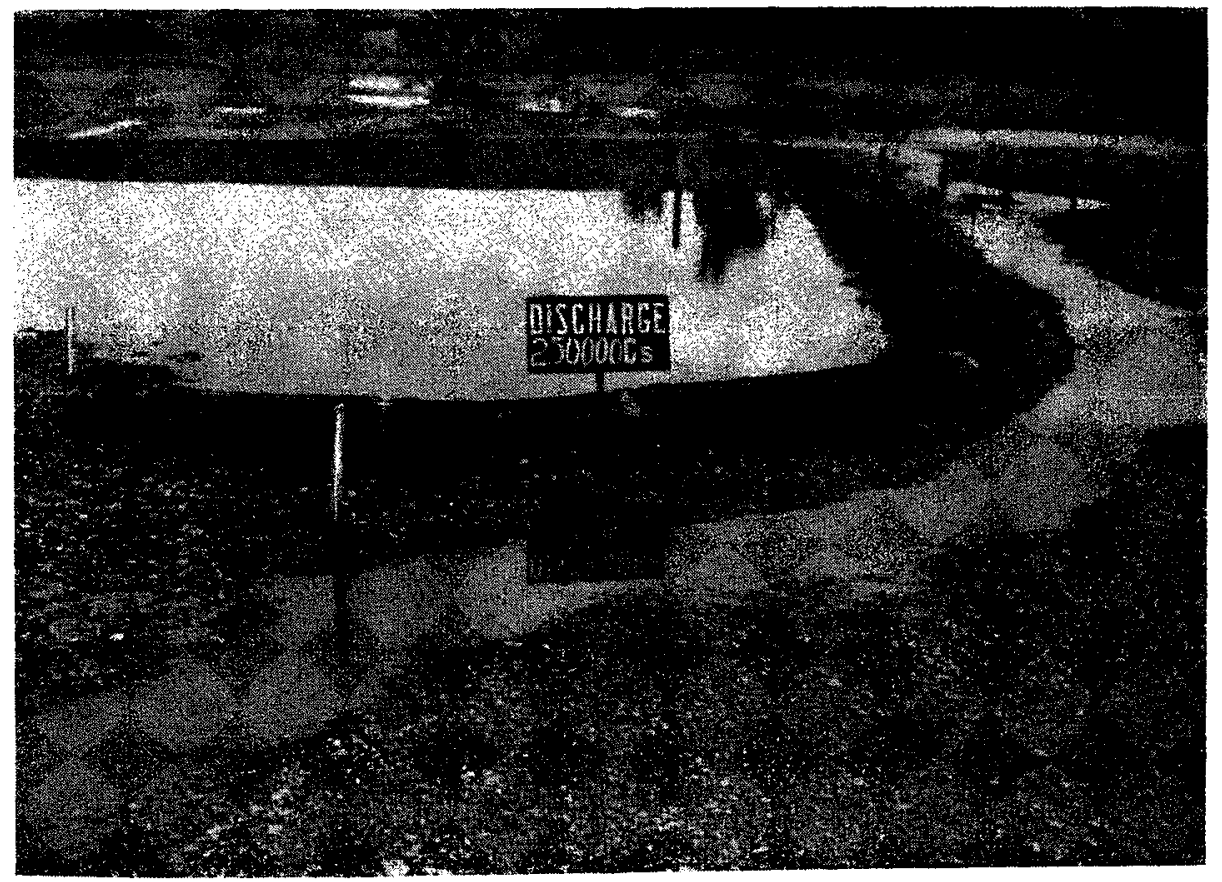

Fik. 10.

Effet de l'érosion

sur la partic courbe

du mur-guideau de gauche.

Erosion caused

in the curved portion of the left guide bank. 
dans le talus tout près du pont principal, provoquait un écoulement parallèle tout le long du talus; on a done décidé de barrer le bras et de le détourner vers la rivière principale.

Bouchure dU BarNaI AU FRANCHISSEMENT DE LA VOIE FERRÉE. - On effectuait cette bouchure à l'aide d'une digue d'approche. On reproduisait sur le modèle différents débits; l'eau montait le Iong de la digue et se mettait à couler parallèlement avant de rejoindre la rivière principale au musoir du mur-guideau de la rive gauche. Ce courant parallèle avait une vitesse de $6,5 \mathrm{ft} / \mathrm{sec}$. $(2 \mathrm{~m} / \mathrm{s}$ ) (voir tableau IV); il fallait donc empierrer et couler du ciment sur toute la longueur du talus entre le point de franchissement du Barnai et le pont; mais cela entraînait de lourdes dépenses d'entretien. De plus, il fallait élever le talus an-dessus du niveau des grandes crues, sur toute sa longueur, jusqu'à Bhangala. Il en résultait de grands frais; aussi le projet fut-il abandonné.

Construction D'Un ensfmble pont-ouvrage DE RÉGLAGE. - A un certain moment, l'idée fut émise qu'il ne fallait pas barrer le Barnaí d'un coup, mais qu'il était préférable de construire un ensemble pont-ouvrage de réglage, où l'on réglerait judicieusement le débit du bras de façon à le colmater graduellement, jusqu'à bouchure complète. Ce projet fut également étudié sur le modèle, mais ne fut pas retenu, et l'on décida finalement de barrer le bras à son origine, c'est-à-dire au point de bifurcation, et de l'envoyer dans la rivière principale à travers le bras jumeau.

4. Emplacement et forme à donner à la digue de dérivation du Barnai.

Tout d'abord, cette digue avait été établie un peu en aval du point de bifurcation, mais on s'aperçut qu'elle avait le défaut de se laisser contourner par la droite. On la déplaça donc plus en amont, jusqu'au point de bifurcation. Sur le côté gauche, on l'ancra à une éminence de cote 892,0 proche du village de Bakranwan. A droite, elle fut accolée à l'îlot Sarkanda (cote $883,0)$, ce qui avait également pour effet d'obturer quelques chenaux s'étendant du bras central jusqu'à pénétrer dans le Barnai. Le faîte de la digue était fixé à la cote 892,0 . Sur la figure 11, on voit quels étaient son emplacement et son alignement.

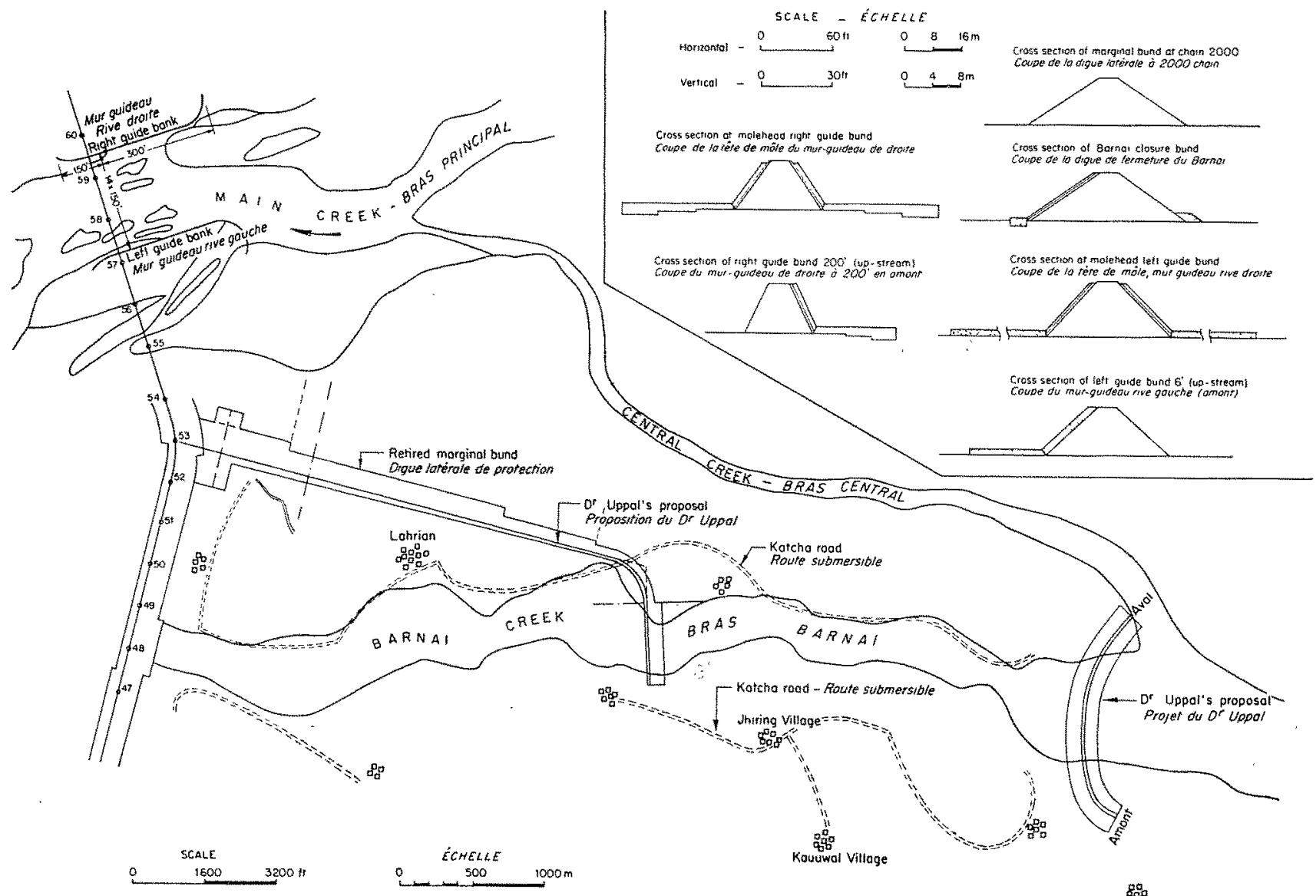

Fic. 11. - Digue de dérivation du Barnai et digue latérale de protection.

Barnai diversion closure bund and the retired marginal bund. 
Pour les besoins de l'irrigation, deux prises munies de vannes furent disposées dans la digue afin d'alimenter respectivement le canal de Basantpur et un second canal. Les prises devaient rester ouvertes toute l'année, sauf au moment des crues du Barnai.

Tous les débits correspondant à ces diverses combinaisons furent réalisés sur le modèle; dans les conditions de débit maximum, le Barnai coulait à $100.000 \mathrm{cs}\left(2.811 \mathrm{~m}^{3} / \mathrm{s}\right)$. On a pu ainsi observer les phénomènes suivants :

a) A mesure qu'augmentait le débit du Barnai, le bras central s'amplifiait et assurait la totalité du débit;

b) Les points de lîlot central et de l'îlot de Sarkanda, qui constituent une partie de l'ouvrage de dérivation, subirent les effets des grandes vitesses d'écoulement et commencèrent à s'éroder. Comme ces points participent au contrôle de l'écoulement, on estima nécessaire de les conserver et de recommander leur protection par un empierrement cimenté, afin qu'ils ne soient jamais emportés;

c) Le niveau maximum de leau contre la digue de fermeture a atteint la cote 890,5 ;

d) Une baisse de niveau de 6,5 pieds $(2 \mathrm{~m}$ ) a été notée entre le Barnai et son bras jumeau, en un point opposé à l'extrémité de la digue de dérivation;

e) La digue de dérivation se trouvant en retrait entre les ilots protégés, s'est trouvée soustraite à l'action du courant parallèle.

5. Choix de l'alignement et du tracé à donner à la digue latérale de protection.

L'examen des essais effectués à cet effet a montré que la digue conforme au second projet donnait de meilleurs résultats et présentait les avantages suivants vis-à-vis des deux autres :

I. Territoire inondé réduit.

II. Réduction au minimum de la longueur des talus devant être surélevés à la cote 861,0 . L'abaissement du talus pouvait être consenti à partir du point Ch. 53000 au lieu des points Ch. 52000 et Ch. 49000 prévus dans les autres projets.

III. Digue latérale très courte.

Afin de prendre une décision définitive au sujet de ces travaux d'endiguement, une conférence d'experts de divers ministères eut lieu le 30 novembre 1950 à la Station de Recherches Hydrauliques de Malakpur.

Les personnes suivantes prirent part aux discussions :
1. Shri Daya Chand Tain, Chief administrative Officer, E.P. Railway.

2. Shri Iqbal Singh the then A.O.E. (Special).

3. Shri H.P. Mathrani, Chief Engineer, Ministry of Transport and Communication, Government of India.

4. Shri S.L. Bazaz, Consultant Ministry of Roads and Transport, Government of India.

5. Shri Kanwar Sain, Chief Engineer, Central Design Office, Government of India.

6. Shri Hukam Singh, Superintending Engineer, P.W.D., Punjab, B \& R Branch.

7. Shri H.H.L. Sethi, Director, Civil Engineering Railway Board, New Delhi.

8. Shri D.C. Sharma, Superintending Engineer, Punjab B \& R Branch.

9. Shri C.L. Handa and

Dr. H:L. Uppal, from Irrigation \& Power Research Institute.

Après étude minutieuse et discussion, les décisions suivantes ont été prises :

1. Le Barnai doit être barré au point où il se détache de la rivière principale;

2. Une nouvelle digue est à établix, à titre de seconde ligne de défense, à mi-chemin entre le point de séparation et le croisement avec la voie ferrée;

3. Rien ne fut décidé au sujet de la digue latérale. Le côté économique de chacun des projets devait être élucidé pour permettre le choix le meilleur et le plus économique.

S'inspirant de ces considérations, et après étude sur modèle des différents projets, les ingénieurs du Chemin de Fer se décidèrent, le 3 octobre 1951, pour le deuxième projet, présenté par le docteur Uppal; ce projet a donc été adopté, séance tenante.

\section{Le projet final.}

Le projet final comprenait les caractéristiques suivantes :

1. Le Barnai est à barrer par une digue de dérivation, à construire au point de bifurcation, a 4 miles $(6,4 \mathrm{~km})$ en amont du talus ferroviaire, les eaux étant détomnées vers la rivière principale par le bras central.

La face amont de la digue devra être pourvue d'un empierrement cimenté, ou bien revêtue de blocs de béton au ciment, pour ne pas céder à l'érosion sous un batillage intense.

Les points de l'îlot du centre et de l'ilot de Sarkanda, ainsi que le musoir aval de la digue, constituant autant de points de contrôle de l'ćcoulement, devront être protégés par des empierrements cimentés, pour ne pas être emportés, quoiqu'il arrive. Une ou deux prises devront être observées dans la digue pour servir à l'irriga- 


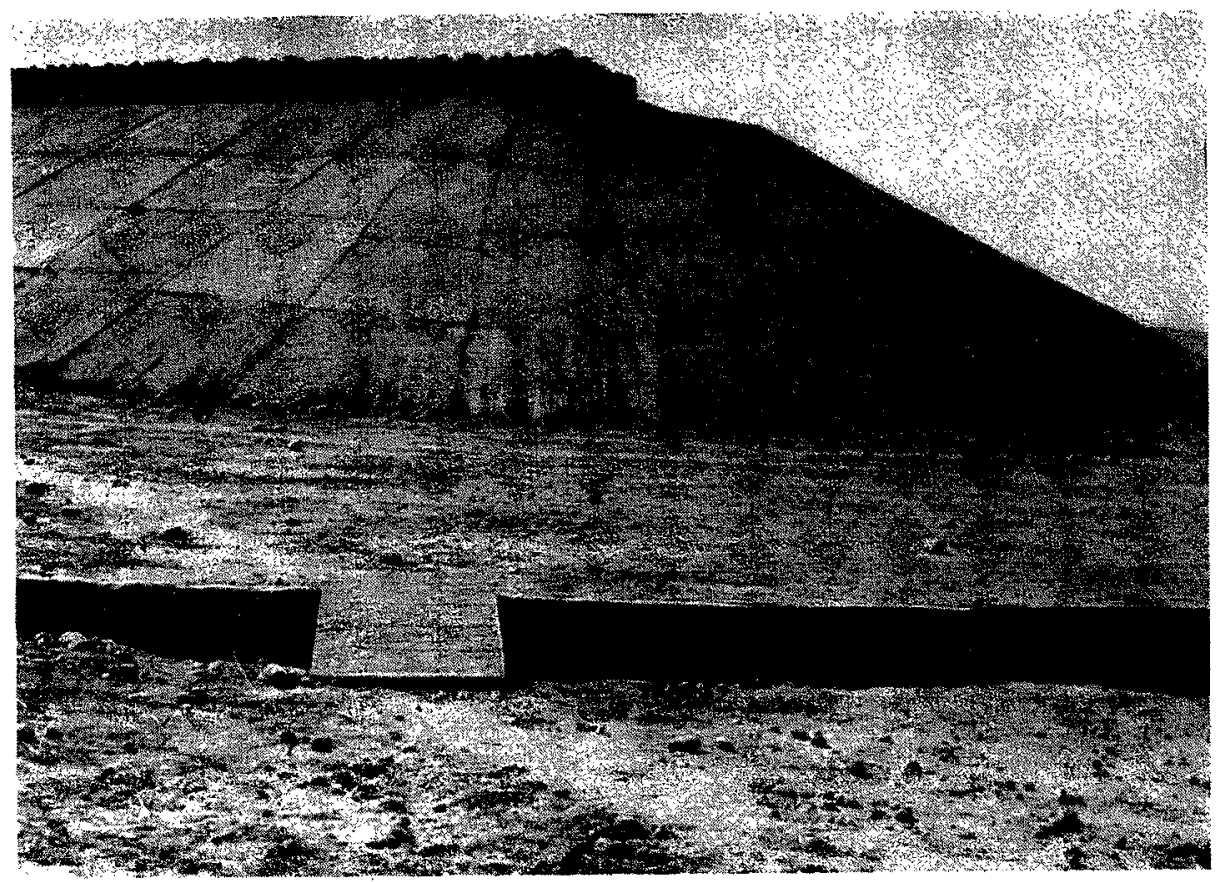

F16, 12.

Musoir aval de la digue de derivation du Barnai.

Downstream mole head of the Barnai closure hund.

tion; elles pourront être maintenues ouvertes toute l'année, sauf aux moments de crue du Barnai.

2. Pour empêcher l'eau proche du pont de s'écouler vers Mukerian et d'y provoquer des dégâts, une digue latérale de protection devra être construite, conforme au projet du docteur Uppal. Elle devra être prolongée en amont et ancrée à Tanda après avoir traversé le Barnai à Lahrian. Dans la digue du Barnai, on devra pré- voir une petite ouverture pour l'eau drainée en cet endroit. Une vue de ces ouvrages, après leur achèvement, est donnée par la figure 11.

\section{Réalisation du projet.}

Le projet a été exécuté sur place par le Ministère des Chemins de Fer. En août 1950, au moment de la crue à Mirthal, la plus forte que la rivière Beas eut jamais à subir et qui correspondait à un débit de $412.000 \mathrm{cs}\left(11.666 \mathrm{~m}^{3} / \mathrm{s}\right)$,
Fir. 13.

Blocs de béton destinés à être appliqués contre les bords et la pointe des îlots.

Cement concrete blocks being laid against the edge and nose of belas.

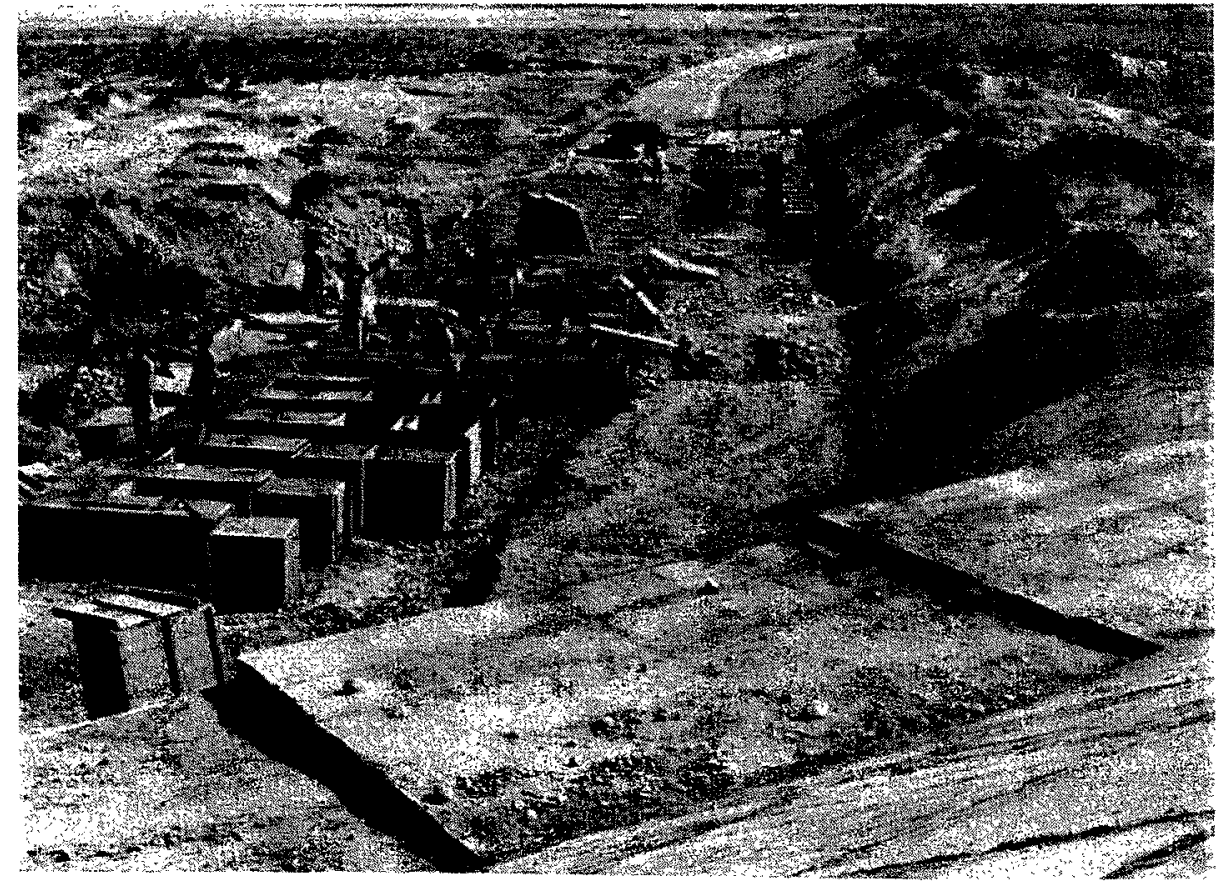


Fig. 14.

Vue de la digue de dérivation du Barnai achevéc.

A niew of the completed Burnat diversion closure bund.

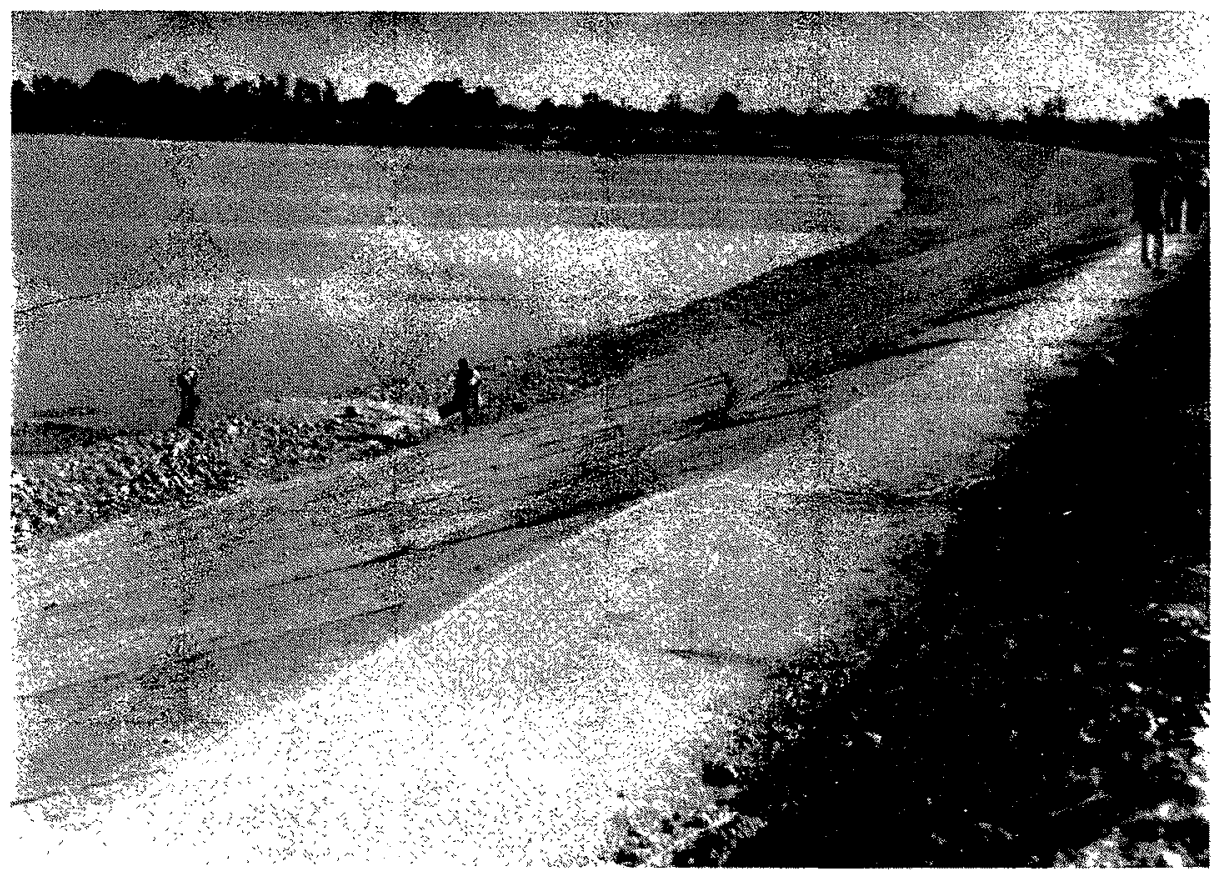

scules les digues d'approche étaient achevées. La digue de dérivation du Barnai et la digue latérale le protection n'étant pas prêtes, l'eau déborda vers Mukerian et causa des dégâts au talus et aux terres basses environnantes. L'événement montra la nécessité urgente des travaux. Aussi les a-l-on entrepris immédiatement et achevés avant la mousson de 1951. La figure 12 montre le musoir aval du Barnai pendant sa construclion. La figure 13 montre la pointe de l'ilot en Irain d'être empierré et cimenlé. La figure 14 donne une vue d'ensemble de la digue après achèvement; celle-ci a dû être protégée par des blocs de béton áu ciment posés contre sa face amont pour ne pas être disloquée par les eaux. La digue latérale de protection fut également achevée avant les crues de 1951.

\section{Comment se comportèrent les ouvrages.}

La digue de dérivation s'est très bien comportée depuis l'achèvement des travaux. Elle a délourné efficacement les eaux du Barnai vers le bras central de la rivière et préservé ainsi les villages de Basantpur, Tanda, Lahrian, Milwen, ete., des effets de la crue. Elle a arrèté toute érosion ultérieure de terres et a provoqué un fort sentiment de sécurité chez les gens des environs, qui se sont remis a la grande culture des arbres fruitiers.

Du fait du judicieux alignement et de l'heureux emplacement de la digue de dérivation par rapport aux pointes empierrées et cimentées de l'ilot central et de l'îlot de Sarkanda, le bras
TABIEAU V

Comparatson des niveaux de l'bau OBSERVÉS SUR LE MODELE ET SUR PLACT

\begin{tabular}{|c|c|c|c|c|c|c|}
\hline \multirow[t]{2}{*}{ 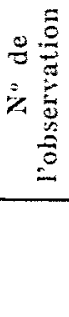 } & \multicolumn{2}{|c|}{$\begin{array}{l}\text { Niveaux } \\
\text { observés sur } \\
\text { le modèle }\end{array}$} & \multicolumn{2}{|c|}{$\begin{array}{c}\text { Niveaux } \\
\text { observés sue } \\
\text { place } \\
\text { pendant les } \\
\text { crues de } 1953\end{array}$} & \multicolumn{2}{|c|}{$\begin{array}{c}\text { Niveaux } \\
\text { observés sur } \\
\text { place } \\
\text { pendant les } \\
\text { crues de } 1955\end{array}$} \\
\hline & $\begin{array}{l}\text { Débits } \\
\text { en es } \\
\left(\mathrm{m}^{\mathrm{B} / \mathrm{s}}\right)\end{array}$ & $\begin{array}{c}\text { Niveau } \\
\text { de } \\
\text { l'cau }\end{array}$ & $\begin{array}{l}\text { Débits } \\
\text { en es } \\
\left(\mathrm{m}^{3} / \mathrm{s}\right)\end{array}$ & $\begin{array}{l}\text { Niveau } \\
\text { de } \\
\text { l'eau }\end{array}$ & $\begin{array}{l}\text { Débits } \\
\text { en es } \\
\left(\mathrm{m}^{3} / \mathrm{s}\right)\end{array}$ & $\begin{array}{l}\text { Niveau } \\
\text { de } \\
\text { l'ean }\end{array}$ \\
\hline 1. & $\begin{array}{r}15,000 \\
(425)\end{array}$ & 836.21 & $\begin{array}{r}15,000 \\
(425)\end{array}$ & 836.15 & $\begin{array}{r}15,333 \\
(434)\end{array}$ & $\ldots$ \\
\hline 2 & $\begin{array}{r}30,000 \\
(850)\end{array}$ & 837.80 & $\begin{array}{r}30,271 \\
(865)\end{array}$ & 837.90 & $\begin{array}{r}30,100 \\
(852)\end{array}$ & 834.73 \\
\hline 3 & $\begin{array}{r}(60,000 \\
(1.390)\end{array}$ & 838.30 & $\begin{array}{r}(62,554 \\
(1.770)\end{array}$ & 838.90 & $\begin{array}{r}(60,237 \\
(1.705)\end{array}$ & 837.63 \\
\hline 4 & $\begin{array}{r}75,000 \\
(2.120)\end{array}$ & 838.50 & $\begin{array}{r}76,487 \\
(2.165)\end{array}$ & 838.60 & $\begin{array}{r}74,125 \\
(2.095)\end{array}$ & 839.50 \\
\hline 5 & $\begin{array}{r}120,000 \\
(3.450)\end{array}$ & 841.60 & $\begin{array}{r}98,586 \\
(2.790)\end{array}$ & 840.60 & $\begin{array}{r}131,75 \mathrm{~d} \\
(3.76 \mathrm{in})\end{array}$ & $841 .(65)$ \\
\hline 6 & $\begin{array}{r}250,000 \\
(7.090)\end{array}$ & 845.30 & $\begin{array}{r}199,023 \\
(5.610)\end{array}$ & 844.000 & $\cdots$ & - \\
\hline
\end{tabular}

* Les chifres entro parentheses sont en mo/s. 
central s'est effectivement dérivé vers la droite et s'est considérablement développé, conformément aux indications du modèle.

Digue ratérale de protection. - Cette digue a également très bien fonctionné. Elle a toujours retenu les eaux sur la longueur limitée prévue de talus ferroviaire (jusqu'au point Ch. 53000), et a empêché l'écoulement de ces eaux vers $\mathrm{Mu}$ kerian. Elle a donc préservé des dégâts le talus de la voie ferrée et les autres ouvrages, ponts, etc., et mis les points bas à l'abri de l'inondation. L'aire comprise entre la digue latérale et le murguideau de la rive gauche a été envahie par d'im- portants dépôts. Toutes ces observations confirment les constatations faites sur le modèle.

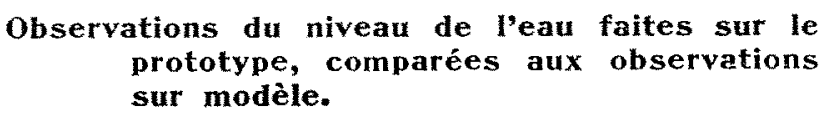

Observations du niveau de l'eau faites sur le prototype, comparées aux observations sur modèle.

Les observations des niveaux au pont ont été effectuées par le Service des Chemins de Fer pendant la mousson de 1951. Comparées à celles qui ont été obtenues sur le modèle, elles révélèrent une coincidence étroite (tableau V) - nouvelle confirmation de la valeur des expériences sur modèle.

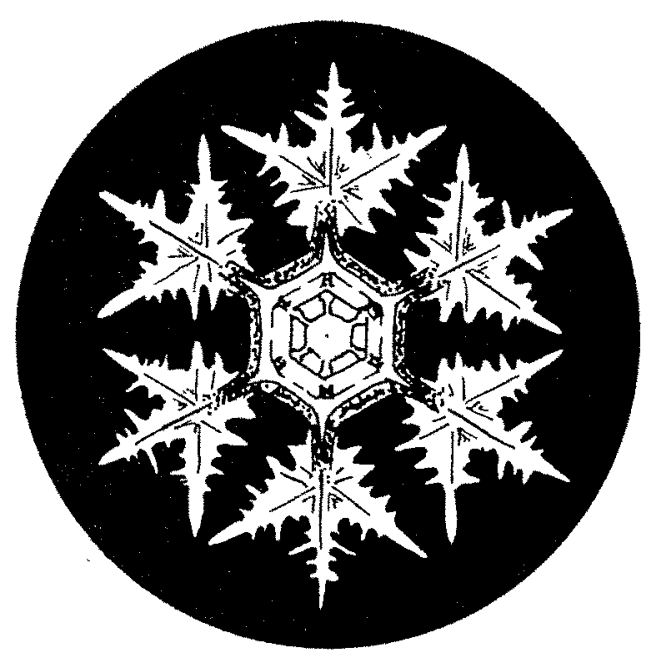




\title{
Training of Beas River for Rail Road Bridge at Mirthal Mukerian-Pathankot Rail Link
}

\author{
IPY H.L. UPPAL \\ DIRECTOR OF MRIGATION AND POWER RESEARCH INSTITUTE OF PUNAAB, AMMITSAR \\ GAJINDER SINGH \\ ASSISTANT RESEARCK OFFICER \\ AND G.P. SHAHANI \\ ENGINEER-IN-CHIEF, GANGA BRIDGE PROJECT (MOKAMEH)
}

(See French text, p. 857, for illustration)

\begin{abstract}
After the partition of country in 1947, and accession of Jammu and Kashmir States to India, a direct Mukerian-Pathankot Rati-Road link was proposed to be constructed. This involved bridging of Beas river at Mirthal. The Beas at Mirthal flowed in a number of creeks and its left bank being much lower than its right bank, it presented certain difflullies in river training and control of floods. A decision had to be taken whether the crecks wert to be trained into the main stream or separats openings were to be provided for them at their crossings. For the proper study of these things several joint inspections of the river were made.
\end{abstract}

\begin{abstract}
A large-scale model of Beas rivers, the proposed bridge and its appurtenant works was constructed at the Hydranlic Research Station, Malalipur and these problems were investigated.

A complete scheme consisting of Barnai diversion closure bund and a retired marginal bund nas evolved. No separate openings were probided for the different creeks of the river at their crossings. The entire scheme was exe. rated and regular obserwations were made on the protolype. A close agreement has been found between the model and the prototype observations.
\end{abstract}

\section{INTRODUCTION}

Before the partition of Punjab, Pathankot was the terminus station for Broad Gauge and starting station for the Narrow Gauge Kangra Valley Railway. It serwed the requirements of Kulu, Dharamsala and Dalhousie out-agencies. The direction of flow of traffic was mostly to and from Lahore. The traffe needs of Jammu and Kashmir Valley were adequately mel by the Rail-heads at Rawalpindi and Jammu. Wilh the partition in 1947, these two Rail-heads fell to Pakistan. With the partition and the accession of the states of Jammu and Kashmir to India, the direction of flow of traffic changed to and from Delhi side. 'The necessity of a shorter and direct route to Pathankol via Mukerian was keenly felt and thus reduce the distance between Jullundur and Pathankot by as much as 44 miles.

Two alternative schemes were prepared. In the first scheme, the link was to be constructed via Gurdaspur, by bridging the combined waters of Beas and Chakki rivers at Chechian sile, near Gurdaspur. In the second scheme the link was proposed to be constructed from Pathankot to Mukerian direct. After detailed considerations, the second scheme was accepted. This scheme however, involved heavy bridging, the principal of these being the one over Beas river at Mirthat $(14 \times 150 \mathrm{ft}$, each) and the other one being over Chakki river at Dhangu near Pathankot. The crossing over the Beas was in the reach of the river where it flowed in a number of channels in a wide khadir of $13,000 \mathrm{fl}$. The river was required lo be trained into a narrow ehannel for this bridge. This was really a diffeult problem and was solved with the help of large scale river models.

\section{The Beas River.}

The river pises in the Himalayas at Beas Kund about 200 miles from Pathankol. It pierces 
Dhaula Dhar range at Larji and traverses a total distance of 390 miles through hills and plains before it joins the Sutlej river at Harike. It is the principal river of Kaugra and Kulu Valleys and has a total catchment area of 7122 sq. miles. Numerous hill torrents such as Binum, Nagal, Ban-Ganga, Gaj, Tara, Chhaunch, ete. join it enroute. Its minimum discharge is 3,500 cusecs which rises to 412,000 cusecs, the maximum flood discharge during mon-soons.

The river emerges out of the hills and enters into the plains at Reh 20 miles upstream of Mirthal. It makes a semicircular loop from Reh to Nowshehra which is eight miles down stream of Mirthal. The river has always sluck to the right bank at Mirthal, which consists of hard clay and does not seem to have been disturbed for several decades. This was one of the reasons for the selection of this site.

Besides the main creek which flows at the extreme right, the river at Mirthal has two other major creeks, the central creek and Barnai creek. These are situated at distances of 2,000 feet and 8,500 feet respectively from the Mukerian end abutment. These are separated by high belas over-grown with thick Sarkanda grass. The belas get submerged only in very high floods. Besides, there are other shoals and belas also fig. 1.

Barnai channel which is now a major creek, did not exist in 1916 and the main river used to flow in the central creek. Barnai was dug only as an inundation irrigation channel of 15 feet width which in the course of time developed into a major creek of the river. It bifurcates from the central creek at a point four miles upstream of the railway embankment. This is now a heavily meandering stream and flows on a steep slope. It has a bed fall of about 40 feet between the point of bifurcation and the railway crossing. It is developing further rapidly and its discharge is also increasing every year. With in two years (1949 and 1950) it had developed from about 20,000 cusecs to about 70,000 cusecs.

Again, the left bank of Beas river at Mirthal is much lower than its right bank; and the country slopes towards Mukerian. Therefore, whenever the river rises, it spills over its left bank and the spill discharge flows towards Mukerian.

\section{The Bed Materiat.}

The bed of the river consists of sand mixed with shingle of 3.0 to 4.0 inches diameter (fig. 2). The bed of Barnai is still coarser. An average diameter of shingle in the bed of this creek is 5.0 to 7.0 inches.

River Slopes. - The bed slope of the main stream is flatter than the bed slopes of the other two creeks.

These are given below:

Channel
Main river.........

It is important to note that the bed of Barnai at the axis of bridge is $7.5 \mathrm{ft}$. higher than the bed of the main river and $5.0 \mathrm{ft}$. higher than the central creek.

River Stages and Discharges. - No regular gauge or discharge observations of the river at Mirthal have been made in the past. In 1950, some observations were carried out by the Railway department, and are given below. In certain cases the corresponding discharges observed at Sujanpur Terrah are also given.

TABIE I

WATER LEVELS IN DIFHEIZENT DISCHARGES OBSERVED AT PROTOTYPE BEFORE THE CONSTRUCTION OF BRIDGE

\begin{tabular}{|c|c|c|}
\hline \multicolumn{2}{|l|}{ PLACE } & $\begin{array}{l}\text { H. F. Levels } \\
\text { in the } \\
\text { main stream }\end{array}$ \\
\hline \multirow{3}{*}{ Upstream of Bridge site } & 17,000 feel. & R.L. 862.4 \\
\hline & 15,000 feet. & R.L. 862.2 \\
\hline & 13,000 feet. . & R.L. 857.3 \\
\hline \multicolumn{2}{|c|}{ At Bridge site $\ldots \ldots \ldots \ldots \ldots \ldots$} & R.L. 847.5 \\
\hline \multirow{3}{*}{$\begin{array}{c}\text { Downstream of Bridge } \\
\text { site } \ldots \ldots \ldots \ldots \ldots\end{array}$} & 2,500 feet. & R.L. 837.7 \\
\hline & 5,500 feet. & R.L. 836.0 \\
\hline & 9,000 feet. & R.L. 834.8 \\
\hline
\end{tabular}

TABLE II

\begin{tabular}{|c|c|c|c|}
\hline \multirow{2}{*}{$\begin{array}{c}\text { River Discharge } \\
\text { at Sujanpur } \\
\text { Terrah }\end{array}$} & \multicolumn{3}{|c|}{ Gauge at Bridge Site } \\
\hline & $\begin{array}{l}\text { H. F. L. of } \\
\text { Creck No. } 1\end{array}$ & $\begin{array}{l}\text { H. F. L. of } \\
\text { Creek No. } 2\end{array}$ & $\begin{array}{l}\text { H. F. L. of } \\
\text { Creek No. } 3\end{array}$ \\
\hline $1,68,600$ cusecs. . & R. I. 838.2 & R. L. 837.82 & R. L. 837.66 \\
\hline $2,50,600$ cusecs. . & R. I. 840.25 & R. L. 839.77 & R. L. 840.45 \\
\hline 70,000 cusecs. & R. L. 836.75 & R. I. 837.00 & R. T. 838.05 \\
\hline
\end{tabular}

\section{Proposals for River Training of Flood Control.}

Since the rail-road bridge had to be constructed over the main stream of the river, the other 
two creeks, Barnai and the central creeks had to be diverted and trained into the main stream or else separate openings had to be provided in the railway embankment at their crossings. Further, with the construction of this bridge and the subsequent reduction in the water-way from $13,000 \mathrm{f}$, the khadir width of the river, to $2,205 \mathrm{ft}$. the overall length of the bridge, the depth of spill would have somewhat increased till such lime the river had scoured its bed and attained the regime conditions. Besides causing damage to the embankment due to parallel flow, it would have caused damages to several villages, towns and to a large area of standing crops on the way. With a view to protect the railway embankment against parallel flow and the country side from inundation it was proposed to construct a marginal bund, suitably sited in a well retired position, away from the attack of the main river. The necessity of this bund was established beyond doubt by the floods of 1950 when quite a number of villages and a large area of standing crops were submerged, 2,000 ft. length of embankment was washed away and quite a number of bridges and appurtenant works were damaged. These damages might not hawe occurred if a retired marginal bund had been constructed and Barnai creek had been closed at its head.

Three alternative proposals were made.

1. In THIS PRoposal, Barnai creek was closed at the railway crossing and the marginal bund was proposed on the left bank of Barnai. It started from Ch. 48,000 of the railway embankment and was tied to the high level ground at R.L. 892.0 near Bakrarwan. The length of the marginal bund was about six and half miles.

2d. Proposal. - In view of the obvious tendency of Barnai creek to develop every year and on account of the serious danger of the river taking evulsions through the openings provided near the bridge it was considered absolutely essential to close the creek either at the head or its railway crossing. The closure at latter of the two alternatives was not feasible since it would induce parallel flow and always cause damages to the railway embankment. Keeping this in view, the diversion at the point of its bifurcation four miles upstream was thought fit as it would develop its sister creek and flow into the river. Accordingly, a Barnai diversion-cum-closure bund was proposed.

In order to prevent the ponded up water from llowing towards Mukerian and damage the embankment and inundate the low lying lands on its way, a retired marginal bund starting from CH. 53,000 of the railway embankment and ending at a high level point at R.L. 861.0 on a bela between Barnai and the Central creek was pro- posed. The length of the bund was about one and half mile and its top was at R.L. 861.0.

3rd Proposal. - In this proposal the alignment of the retired marginal bund was more or less the same as the alignment in the second proposal. This was however, extended upstream and after crossing Barnai between the villages of Lahrian and Tanda it was tied to the high level ground at R.L. 892.0 near the village Bakrarwan. The length of this bund was about six miles. For this proposal to succeed it was essential to close the Barnai creek at its head. The proposals are shown in fig. 3 .

All these presented quite a difficult and complicated problems. These were discussed in a series of conferences attended by representatives of different departments. After all these discussion, the railway board referred it to the Direclor, Irrigation and Power Research Institute, Punjab, Amritsar, for investigations by means of models, at the Hydraulic Research Station, Malakpur.

\section{Statement of Problems.}

The following problems were required to be investigated on the models :-

1. Study of the behaviour of the river and determination of the maximum water levels at the bridge, nose and tail end of guide banks, against the railway embankment, in the still water pockets, etc., in the maximum designed discharge of 420,000 cusecs.

2. Study of the conditions of flow along the guide banks and observation of afflux at the bridge.

3. Whether Barnai a major creel of the river should be closed and diverted into the main stream or it should be kept running and a separate bridge provided for it at its railway crossing. If it is to be closed, should it be closed at the point of its bifurcation from the central creek or it should be closed at the railway crossing.

4. Investigation of the position and design of Barnai diversion closure bund.

5 . Investigation of the most suitable alignment and design of the retired marginal bund.

\section{Data Available.}

The following data was obtained.

1. Survey plan of the river for a length of four miles upstream and two and a half miles downstream of the bridge site.

2. River sections $500 \mathrm{ft}$, apart up to one and a half mile and $1,000 \mathrm{ft}$. apart up to four miles 
on the upstream side. Similarly on the downstream side sections $500 \mathrm{ft}$. apart upto half a mile and $1,000 \mathrm{ft}$. apart upto two and half miles.

3. Tentative design of the bridge and the appurtenant works.

4. Piver sections and survey plans of the l'ara and Chhaunch khuds.

5 . Old contour survey plan of the area on the left side of the bridge.

6. Bed samples, isolated river discharge and gauges observed at Mirthal in 1950.

7. Aerial survey map of the river.

8. Old Irrigation map.

\section{Examination of the Data.}

The data available was examined and the following observations were made :

(i) A ridge exists between Barnai and the Central creek and extends upto 6,000 ft. from the railway embankment. Except for this reach where the levels of the ridge and the ground are low, the levels of the ridge are higher than any of the highest flood levels and therefore it never gets submerged even in high floods.

(ii) The natural surface above one mile upstream of the railway embankment slopes from left to right and at a point four miles upstream of the bridge there is a difference of 24 feet in the flood levels.

(iii) At Mirthal, the left bank of the river is much lower than the right bank. Floods spill heavily over its left bank and inundates the entire low lying country side towards Mukerian.

(iv) Barnai is larger than the central ereek. In the bed, there is a fall of 35 to 40 feet in a length of about four and a half miles. The bed of the main river has a drop of only 15 to 17 feel in the same length.

\section{Model Investigations.}

The Monel. - A model of the Beas river for a length of five miles upstream and two miles downstream, entire width of the khadir and the creeks, the proposed Railway bridge and its appurtenant works was constructed at the Hydraulic Research Station, Malakpur. The river bed was moulded with a mixture of sand and gravel of half inch diameter. Major Khuds such as Tara and Chhaunch which join the river from the right side were also simulated. Gauges were fixed at important points on the model to read water-levels in different discharges. Completed model is shown in fig. 4 .
Scales. - The following scales were adopted :

Horizontal-model to prototype $1: 90$.

Vertical-model to prototype. $1: 30$.

Exaggeration ............ 3.

Discharge scale.......... 1 : 14788.0.

Area under the model..... 270,000 sq. ft.

Actual discharge run on the

model ............ 28.4 cusecs.

Sand and shingle grade used

on the model.......... 0.5 inch diameter.

OPERATION OF THE MODEL. - The model could not be proved since the data required for carrying out these tests was not available. However, it was considered that it was not essential to have the proving tests done for this work. The following studies were made :-

Stady No. 1. - Study of the behaviour of the river and determination of the maximum water levels at the bridge, nose and tail end of guide banks , against the railway embankment, in the still water pockets, etc., in the maximum designed discharge of 420,000 cusecs.

The model was run with discharge varying from 15,000 cusecs to a maximum designed discharge of 420,000 cusecs. Major part of the discharge was earried by the Beas river. In the maximum flood discharge of 420,000 cusecs, Barnai carried a discharge of 75,000 cusecs. In this maximum flood discharge the maximum water level of R.L. 858.2 and R.L. 857.3 were observed in the left and right still water pockets and a maximum level of R.L. 858.0 was observed against the approach bank. Maximum water levels recorded at the nose and tail of guide bank were R.L. 856.75 and R.L. 847.25 respectively. Water levels recorded at all the important points are given in Table III.

It was observed that the river upto a discharge of 50,000 cusecs did not spill over its hanks. In higher discharges it spilled over its banks and the spill water headed up against the Railway embankment and formed still water pockets, behind the guide banks. The heading up extended to different distances in different discharges, table IV.

The Sarkanda belas and the ridge between Barnai and the central creek was not over-tapped. Only a portion of the land upto $6,000 \mathrm{feel}$, upstream of the embankment was submerged. Bcla between creek No. 2 and the main river was partially submerged.

Study No. 2. - A study of the conditions of flow along the guide hanks and observation of aftux at the bridge. 
TABLE III

\begin{tabular}{|c|c|c|c|c|c|c|c|c|c|c|}
\hline \multirow[b]{3}{*}{ Sr. PoINT of No. observation } & \multicolumn{10}{|c|}{ DISCHARGES } \\
\hline & \multicolumn{2}{|c|}{$75,000 \mathrm{cs}$} & \multicolumn{2}{|c|}{$120,000 \mathrm{cs}$} & \multicolumn{2}{|c|}{$250,000 \mathrm{cs}$} & \multicolumn{2}{|c|}{$360,000 \mathrm{cs}$} & \multicolumn{2}{|c|}{$420,000 \mathrm{cs}}$. \\
\hline & 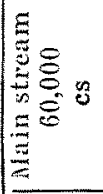 & 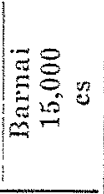 & 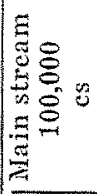 & 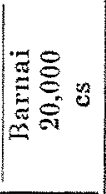 & 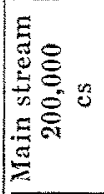 & 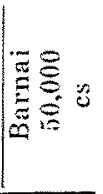 & 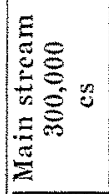 & 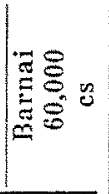 & 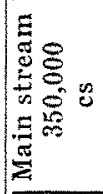 & 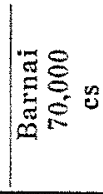 \\
\hline 1. $6,000 \mathrm{ft} . \mathrm{u} / \mathrm{s}$ of Bridge $\ldots \ldots \ldots \ldots$ & \multicolumn{2}{|c|}{845.10} & \multicolumn{2}{|c|}{846.90} & \multicolumn{2}{|c|}{852.13} & \multicolumn{2}{|c|}{856.26} & \multicolumn{2}{|c|}{857.90} \\
\hline 2. At the Bridge $\ldots \ldots \ldots \ldots \ldots \ldots \ldots$ & \multicolumn{2}{|c|}{839.50} & \multicolumn{2}{|c|}{841.20} & \multicolumn{2}{|c|}{846,0} & \multicolumn{2}{|c|}{849.70} & \multicolumn{2}{|c|}{849.80} \\
\hline 3. $2,500 \mathrm{rt} . \mathrm{d} / \mathrm{s}$ of Bridge $\ldots \ldots \ldots \ldots$ & \multicolumn{2}{|c|}{835.50} & \multicolumn{2}{|c|}{836.40} & \multicolumn{2}{|c|}{839.5} & \multicolumn{2}{|c|}{842.65} & \multicolumn{2}{|c|}{843.60} \\
\hline 4. Nose of Guide Banks ............ & \multicolumn{2}{|c|}{843.0} & \multicolumn{2}{|c|}{845.5} & \multicolumn{2}{|c|}{851.8} & \multicolumn{2}{|c|}{855.5} & \multicolumn{2}{|c|}{856.75} \\
\hline 5. Tail of Guide Banks........ & \multicolumn{2}{|c|}{838,40} & \multicolumn{2}{|c|}{839.85} & \multicolumn{2}{|c|}{844.25} & \multicolumn{2}{|c|}{846.60} & \multicolumn{2}{|c|}{847.25} \\
\hline 6. Still water Pocket-Left. ........... & \multicolumn{2}{|c|}{845.5} & \multicolumn{2}{|c|}{847.0} & \multicolumn{2}{|c|}{852.5} & \multicolumn{2}{|c|}{856.5} & \multicolumn{2}{|c|}{858.2} \\
\hline 7. Still water Pocket-Right.......... & $84{ }^{\circ}$ & 3.5 & 84 & 5.6 & 851 & & 855 & 5.5 & 85 & 7.2 \\
\hline $\begin{array}{l}\text { 8. Against embankment at } 2,000 \mathrm{ft} \text {, left } \\
\text { of bridge. } \ldots \ldots \ldots \ldots \ldots \ldots \ldots \ldots \ldots\end{array}$ & 845 & 5.3 & 84 & 0.5 & 852 & & 856 & & 85 & 7.8 \\
\hline 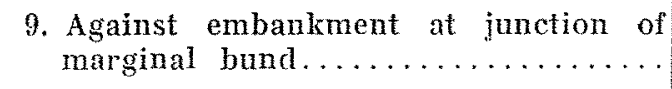 & 845 & 5.0 & 84 & 6.5 & 852 & 2.0 & 856 & & 857 & 7.70 \\
\hline 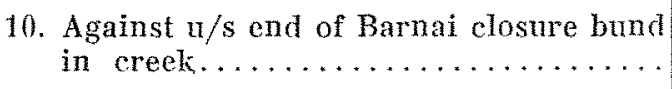 & 879 & .0 & 87 & 9.75 & 880 & & 882 & & 884 & 4.3 \\
\hline 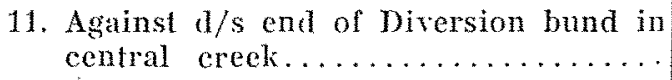 & 878 & 3.1 & 87 & 8,50 & 879 & & 880 & & 880 & 0.2 \\
\hline
\end{tabular}

TABLE IV

SHOWING VeloctTy OF PARALIEL FIOW AND THE EXTENT OF MEADING UP AGANST THE RAILWAY EMBANKMENT

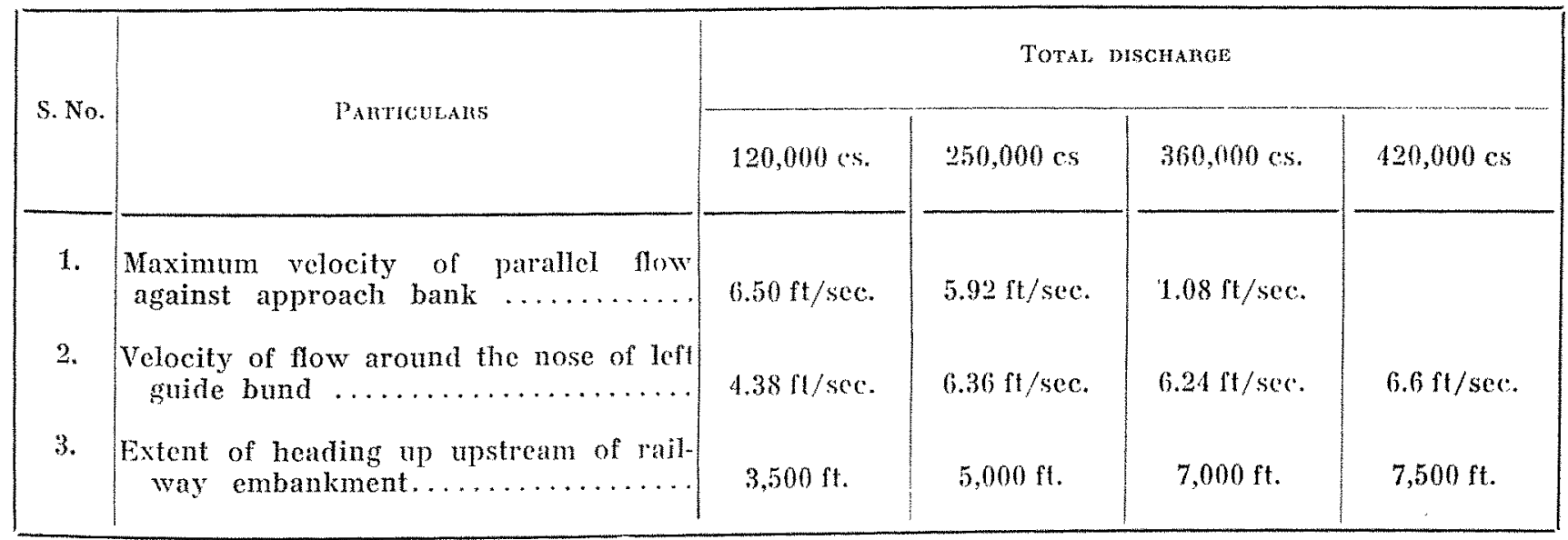

The current directions and velocities were observed both along the guide banks and across the river at the bridge. It was observed that the flow between the guide banks was uniform. Figures 5 to 8 . Maximum velocity of flow along the guide bank was of the order of $16.0 \mathrm{ft} / \mathrm{sec}$. 
Left Guide Bank. - In low discharges there was no action on any of the guide banks. In high discharges, however, the main current hit the left guide bank at a point about 450 feet from its nose fig. 9 and caused heavy action. Fig. 10. Its upstream length measuring between 2,300 feet and 3,000 feet from the embankment came under action, action being very severe along the curved portion of the bund.

Right GUide Bank. - The main current met the right guide bank downstream of the Bell bund portion, its upper part always stood in a pool of still water. Bell bund portion of this guide bank came into action only when the floods in Beas river and Chhaunch Khud synchronised.

There was an afflux of about two feet of water which was rather high on account of the rapid at the bridge site.

Study No. 3. - Whether Barnai a major creek of the river should be closed and diverted into the main stream or it should be kept running and a separate bridge provided for it at its railway crossing. If it is to be closed should it be closed at the point of its bifurcation from the central creek or it should be closed at the railway crossing.

Barnai creek was developing rapidly every year. This tendency of the creek was confirmed on the model also. Under these circumstances, the extent of openings to be provided in the embankment to keep Barnai running could not be exactly determined. Besides, on account of the fear of river taking evulsion through the additional openings it was not considered suilable to provide any big openings near the major bridge. A large opening in the embankment close to the main bridge induced a parallel flow along the railway embankment. It was thus decided to close it and divert it into the main river.

BahNaI Closed at THE RAILWAY Crossing. Barnai was closed at its railway crossing by the approach bank. The Model was run with different discharges. The water headed up against the approach bank and started flowing parallel to it before it joined the main river at the nose of left guide hank. The velocity of the parallel flow was 6.5 feet per second. Table IV. This necessitated stone pitching and cement grouting, of the entire length of the embankment from Barnai crossing to the bridge and involved heavy recurring maintenance expenses. It also necessitated raising of the entire length of the embankment upto Bhangala to above high flood level. This made the proposal very expensive and was thus abandoned.

Construction of REgUlator-CUM-BRIDGE. At one occasion it was suggested that Barnat should not be closed in one stage. A regulatorcum-bridge with a raised crest should be constructed and by judicious regulation of its discharge at this regulator, it should be gradually silted up and then finally closed. This proposal was also examined on the model but was not found suitable. It was thus decided to close it at the head i.e. the point of its bifurcation and divert it into the main river through its sister creek.

Study No. 4. - An investigation of the position and design of the Barnai diversion closure bund.

Al first the closure bund was constructed a little downstream of the point of its bifurcation. It was observed that the bund did not function satisfactorily. It outflanked on the right. The closure bund was then shifted upstream and was constructed at the point of its bifurcation. On the left it was tied to a high level ground at R.L.892.0 near the village Bakranwan. On the right it was tied to Sarkanda bela at point at R.L. 883.0. This also plugged small depressions running from the central creek into Barnai. Top level of the bund was kept at R.L. 892.0. The position and alignment of the bund is shown in fig. 11.

Two controlled culverts were provided in the bund for irrigation purposes. One was meant to feed Basantpur channel and the other was meant for the other irrigation channel. The culverts were to be kept open all the year excepting when Barnai was in floods.

All the different discharges were run on the model. In the maximum designed discharge, Barnai carried a discharge equivalent of 100,000 cusees. In these tests it was observed that :-

1. As the discharge in Barnai increased the central creek corresponding developed and took care of the entire discharge.

2. Noses of the central bela and the Sarkanda bela which form part and parcel of the diversion bund came under action of high velocity current and started eroding. Since these form the control points it was considered essential to maintain them and was recommended that the noses as well as these parts of the belas should be protected by grounted stone pitching so that they were not washed away under any circumstances.

3. Maximum water level against the closure bund was observed at R.L. 890.5 .

4. A drop of 6.5 feet in water level was observed between Barnai and its sister creeks at a point opposite the end of diversion bund.

5 . Diversion bund being in a set back position and between the protected islands no parallel flow took place against it. 
Study No. 5. - An investigation of the most suitable alignment and design of the retired marginal bund.

Examination of results of the tests indicated that the marginal bund of second proposal funclioned better and had the following advantages over the other two :-

(i) It brought a minimum area of land under submergence.

(ii) A minimum length of the embankment had to be raised to the formation level of R.L. 861.0. The level of the embankment could be lowered from $\mathrm{Ch}$. 53,000 as against Ch. 52,000 and Ch. 49,000 in the case of other proposals.

(iii) The length of the marginal bund was very short.

To lake a final decision upon the design of these training works a conference of experts from different departments was held on 30 th November, 1950 at the Hydraulic Research Station Malakpur.

Among those who took part in the discussions were :-

1. Shri Daya Chand Jain, Chief Administrative Officer, E.P. Railway.

2. Shri Iqbal Singh the then A.O.E. (Special).

3. Shri H.P. Mathrani, Chief Engineer, Ministry of Transport and Communication, Government of India.

4. Shri S.L. Bazaz, Consultant Ministry of Roads and Transport, Government of India.

5. Shri Kanvar Sain, Chief Engineer, Central Design Office, Government of India.

6. S. Hukam Singh, Superintending Engineer, P.W.D., Punjah, B \& R Branch.

7. Shri H.K.L. Sethi, Director, Civil Engineering Railway Board, New Delhi.

8. Shri D.C. Sharma, Superintending Engineer, Punjab B \& R Branch.

9. Shri C.L. Handa and Dr. H.L. Uppal from Irrigation \& Power Research Institute.

After a careful study of these, a discussion was held and following decisions were taken :-

1. Barnai should be closed at the point of its take of from the main river.

2. As a second line of defence, another bund may be constructed half way between the point of its ofl take and the railway crossing.

3. No definite decision could be taken about the marginal bund. It was suggested that the economies of the different proposals should be worked out and the one which appeared to be more sound and economical should be adopted.
Keeping these in view and studying the behaviour of different proposals on models on 3rd October, 1951. Railway Engineering Officers decided upon the second proposal, made by Dr. Uppal which was adopted at site.

\section{The final Scheme: -}

The final scheme consisted of the following items :-

1. Barnai should be closed by the construction of a diversion closure bund. It should be constructed at the point of its bifurcation four miles upstream of the railway embankment and the flow diverted into the main river through the central creek.

The upstream face of the closure bund should be stone pitched and cement grouted or it should be laid in cement concrete blocks so that it does not erode due to heavy wave wash.

The noses of the central bela and the Sarkanda bela as also the downstream nose of the bund which forms the control points should be protected by grouted stone pitching so that they do not wash away under any circumstances. One or two culverts may be provided in the bund for irrigation purposes. The culverts may be kepl open all the year excepting when the Barnai is in floods.

2. To prevent the water at the bridge from flowing towards Mukerian and cause damages, a retired marginal bund should be constructed according to Dr. Uppal's purposals. It should be extended further upstream and after crossins Barnai et Lahrian it should be tied at Tanda. For the disposal of the local drainage water some small opening may be kept in the Barnai bund. The final scheme of these works is shown in fig. 11.

\section{Execution of the Scheme : -}

This scheme was executed at site by the Railway Department. Till August, $1950^{\circ}$ when the Beas river at Mirthal experienced unprecedented floods of 412,000 cusecs the approach banks alone had been completed. The Barnai diversion closure bund and the retired marginal bund could not be completed as a result of which the flow of headed up flood water took place towards Mukerian and caused damages both to the embankment as well as to the low lying area. This established beyond doubt the imporlance and urgency of these works. The construction of these was taken up in hand immediately and were completed before the monsoons of 1951. Fig. 12. shows the downstream mole head of Barnai bund under construction. Fig 13 shows the edge of the hela being stone pitched and grouted. 
General view of the completed bund is shown in fig. 14. To protect it against wave wash cement concrete blocks have been laid on the upstream face of the bund. The retired marginal bund was also completed before the floods of 1951 .

\section{Behaviour of the works.}

Since the construction of these works the Barnai diversion bund has functioned very well. It has successfully diverted the waters of Barnai into the central creek into the main river. It has thus saved the villages of Basantpur, Tanda, Lahrian, Milwan, etc., from the ravages of floods. If has stopped the further erosion of lands and has created a sense of great security in the minds of these people and they have once again started growing big fruit gardens.

On account of the suitable alignment and positioning of the diversion bund with respect to the pitched and cement grouted noses of central and Sarkanda belas, as observed on the model, the central creek has shifted to the right at the prototype also. It has also developed considerably. This too was observed on the model.

RETRED MARGINAL BUND. - The retired marginal bund has functioned very well. It has always held up the ponded up flood water within the length of embankment upto Ch. 53,000 and has prevented the flow of this water towards Mukerian. Thereby it has saved the railway embankment and other works such as bridges, etc., from damages and the low lying country side from inundation. The area within the marginal bund and the left guide bank has silted up considerably. All these observations confirm the findings of the models.

\section{Observations of water level at the prototype and their comparison with those obtained on the model.}

Observations of water levels at the bridge were made by the railway department during the monsoon of 1951. These were compared with those observed on the model close agreement was found between the two. Table $V$. This further confirmed the findings of the model experiments.

\section{TABLE V}

COMPARATIYE STATEMENT OF WATER LEVELS

\begin{tabular}{|c|c|c|c|c|c|c|}
\hline \multirow{2}{*}{$\begin{array}{l}\text { Sr. } \\
\text { No. }\end{array}$} & \multicolumn{2}{|c|}{$\begin{array}{l}\text { Water levels } \\
\text { observed } \\
\text { on the model }\end{array}$} & \multicolumn{2}{|c|}{$\begin{array}{l}\text { Water levels } \\
\text { observed on } \\
\text { the prototype } \\
\text { in } 1953 \text { floods }\end{array}$} & \multicolumn{2}{|c|}{$\begin{array}{l}\text { Water levels } \\
\text { observed on } \\
\text { the prototype } \\
\text { in } 1955 \text { floods }\end{array}$} \\
\hline & $\begin{array}{l}\text { Dis- } \\
\text { charge } \\
\text { in } \\
\text { cusecs }\end{array}$ & $\begin{array}{l}\text { Water } \\
\text { level }\end{array}$ & $\begin{array}{l}\text { Dis- } \\
\text { charge } \\
\text { in } \\
\text { cusecs }\end{array}$ & $\begin{array}{l}\text { Water } \\
\text { level }\end{array}$ & $\begin{array}{l}\text { Dis- } \\
\text { charge } \\
\text { in } \\
\text { cusecs }\end{array}$ & $\begin{array}{l}\text { Water } \\
\text { level }\end{array}$ \\
\hline 1. & 15,000 & 836,21 & 15,000 & 836.15 & 15,333 & - \\
\hline 2. & 30,000 & 837.80 & 30,271 & 837.90 & 30,100 & 834.73 \\
\hline 3. & 60,000 & 838.50 & 62.554 & 838.90 & 60,237 & 837.63 \\
\hline 4. & 75,000 & 838.50 & 76,487 & 838.60 & 74,125 & 839.00 \\
\hline 5. & 120,000 & 841.60 & 98,586 & 840.60 & 131,734 & 841.65 \\
\hline 6. & 250.0100 & 845.36 & 199,023 & 844.00 & - & - \\
\hline
\end{tabular}

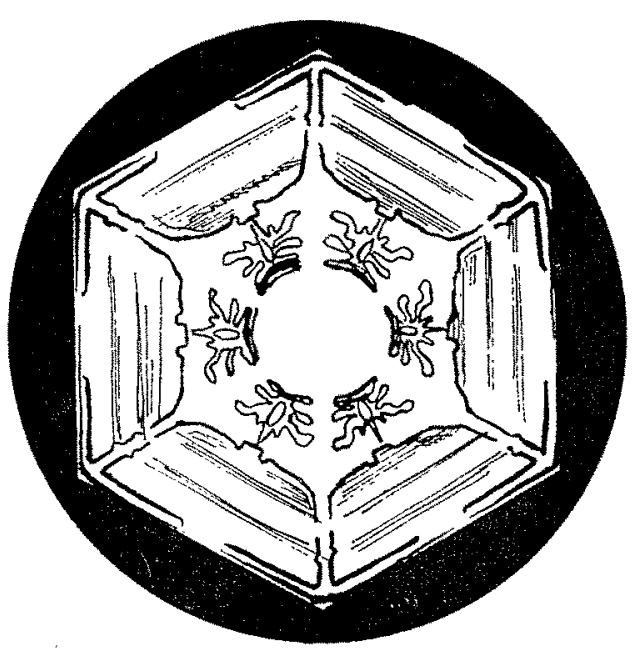

